\title{
Clinical Perspectives and Trends: Microperimetry as a Trial Endpoint in Retinal Disease
}

\author{
Yesa Yang ${ }^{a, b}$ Hannah Dunbar ${ }^{a, b}$ \\ ${ }^{a}$ UCL Institute of Ophthalmology, University College London, London, UK; ${ }^{b}$ Moorfields Eye Hospital NHS \\ Foundation Trust, London, UK
}

\section{Keywords}

Microperimetry $\cdot$ Fundus-related perimetry $\cdot$ Fundus-

automated perimetry Endpoints - Outcome measures

\begin{abstract}
Endpoint development trials are underway across the spectrum of retinal disease. New validated endpoints are urgently required for the assessment of emerging gene therapies and in preparation for the arrival of novel therapeutics targeting the early stages of common sight-threatening conditions such as age-related macular degeneration and diabetic macular oedema. Visual function measures are likely to be key candidates in this search. Over the last 2 decades, microperimetry has been used extensively to characterise functional vision in a wide range of retinal conditions, often detecting subtle defects in retinal sensitivity that precede visual acuity loss and tracking disease progression over relatively short periods of time. Given these appealing features, microperimetry has already been adopted as an endpoint in interventional studies, including multicentre trials, on a modest scale. A review of its use to date shows a concurrent lack of consensus in test strategy and a wealth of innovative disease and treatment-specific metrics which may show promise as clinical trial endpoints. There are practical
\end{abstract}

karger@karger.com www.karger.com/oph

Karger $\stackrel{\text { ' }}{5}$

GOPEN ACCESS
(C) 2021 The Author(s)

Published by S. Karger AG, Basel

This is an Open Access article licensed under the Creative Commons Attribution-NonCommercial-4.0 International License (CC BY-NC) (http://www.karger.com/Services/OpenAccessLicense), applicable to the online version of the article only. Usage and distribution for commercial purposes requires written permission. considerations to consider for its use, but these have not held back its popularity and it remains a widely used psychophysical test in research. Endpoint development trials will undoubtedly be key in understanding the validity of microperimetry as a clinical trial endpoint, but existing signs are promising.

(C) 2021 The Author(s)

Published by S. Karger AG, Basel

\section{Introduction}

Change in visual function is the US Food and Drug Administration's (FDA) recommended primary endpoint for trials assessing the effect of new therapeutics for ocular conditions [1]. High-contrast best-corrected visual acuity (BCVA) is the only generally accepted visual function endpoint by regulators and payers. Change in BCVA, specifically a loss or gain of 15 or more Early Treatment Diabetic Retinopathy Study (ETDRS) letters, has been successfully adopted in large landmark multicentre clinical trials in ophthalmology over the last decades [2-5]. However, its value in assessing functional deficits in early disease and tracking small but important amounts of progression is limited [6-8]. 
Table 1. Summary of characteristics of commercially available microperimeters

\begin{tabular}{|c|c|c|c|c|c|}
\hline & Nidek MP-1/MP-1S & Nidek MP-3/MP-3S ${ }^{a}$ & MAIA/S-MAIA ${ }^{\mathrm{a}}$ 2nd generation & Optos OCT/SLO & Compass \\
\hline $\begin{array}{l}\text { Background luminance, } \\
\mathrm{cd} / \mathrm{m}^{2}\end{array}$ & $\begin{array}{l}\text { Mesopic: } 1.27 \\
\text { Scotopic: } 0.0025\end{array}$ & $\begin{array}{l}\text { Photopic: } 10 \\
\text { Mesopic: } 1.27 \\
\text { Scotopic: } 0.00095\end{array}$ & $\begin{array}{l}\text { Mesopic: } 1.27 \\
\text { Scotopic: }<0.0001\end{array}$ & Photopic: 10 & Photopic: 10 \\
\hline $\begin{array}{l}\text { Maximum stimulus } \\
\text { intensity, } \mathrm{cd} / \mathrm{m}^{2}\end{array}$ & $\begin{array}{l}\text { Mesopic: } 128 \\
\text { Scotopic: } 0.25\end{array}$ & $\begin{array}{l}\text { Photopic: } 3183.1 \\
\text { Mesopic: } 319.58 \\
\text { Scotopic: } 0.096\end{array}$ & $\begin{array}{l}\text { Mesopic: } 318 \\
\text { Scotopic: } 2.54^{\mathrm{b}}\end{array}$ & 125 & $3,183.1$ \\
\hline Dynamic range, $\mathrm{dB}$ & $0-20$ & $\begin{array}{l}0-34 \text { (photopic and mesopic) } \\
0-24 \text { (scotopic) }\end{array}$ & $0-36$ & $0-20$ & $0-50$ \\
\hline Fundus field of view & $45^{\circ}$ & $45^{\circ}$ & $36^{\circ}$ & $29.7^{\circ}$ & $60^{\circ}$ \\
\hline Fundus image & $\begin{array}{l}\text { B\&W IR (live feedback) } \\
\text { Colour (results display) }\end{array}$ & $\begin{array}{l}\text { B\&W IR (live feedback) } \\
\text { Colour (results display) }\end{array}$ & B\&W SLO & B\&W SLO & Colour, IR, red-free \\
\hline Fundus image resolution & $\begin{array}{l}768 \times 576 \text { pixels }(B \& W) \\
1,392 \times 1,038 \text { pixels }(\text { colour })\end{array}$ & $\begin{array}{l}768 \times 576 \text { pixels }(B \& W) \\
4,290 \times 2,800 \text { pixels }(\text { colour })\end{array}$ & $1,024 \times 1,024$ pixels & $512 \times 512$ pixels & $2,592 \times 1,944$ pixels \\
\hline Threshold strategy & $\begin{array}{l}4-2 ; 4-2-1 \text { staircase, and } \\
\text { others including manual }\end{array}$ & $4-2 ; 4-2-1$ staircase & $\begin{array}{l}\text { 4-2 staircase } \\
\text { Suprathreshold tests }\end{array}$ & $\begin{array}{l}4-2 ; 4-2-1 \\
\text { Staircase and others } \\
\text { including suprathreshold }\end{array}$ & 4-2, ZEST \\
\hline Stimulus duration, ms & $100-2,000$ & 100,200 & 200 & 200,300 & 200 \\
\hline Stimulus size & Goldmann I-V & Goldmann I-V & Goldmann III & Goldmann I-V & Goldman III \\
\hline Normative data & $\begin{array}{l}\text { Provided for mesopic } \\
\text { (local defect maps) }\end{array}$ & Absent & $\begin{array}{l}\text { Provided for standard grid use in } \\
\text { mesopic (macular integrity index) }\end{array}$ & Absent & Provided \\
\hline Fixation tracking speed, $\mathrm{Hz}$ & 25 & 30 & 25 & 8 & 25 \\
\hline Biofeedback training & Yes & Yes & Yes & No & No \\
\hline Importing of images & Yes (images and OCT) & No & No & Yes (OCT) & No \\
\hline
\end{tabular}

B\&W, black and white; IR, infrared; ZEST, zippy estimation by sequential testing; SLO, scanning laser ophthalmoscope.

a References to scotopic features relate to the scotopic version of the device.

${ }^{\mathrm{b}}$ Units based on scotopic luminosity function.

The need for new, validated endpoints in both acquired and inherited retinal disease (IRD) has been widely discussed [9-11], with endpoint development clinical studies currently underway in early and intermediate agerelated macular degeneration (AMD) [12-14], Stargardt disease [8], and retinitis pigmentosa [15]. An ideal endpoint would be capable of being easily and frequently measured; be repeatable with minimal measurement and ascertainment error; be sensitive to change over time and treatment effect; have clinical relevance and be meaningful to patients $[6,16]$. Even if these features were confirmed in a laboratory setting, they must also hold across large, international multicentre clinical trial settings if an endpoint is to be truly expedient at assessing novel therapeutics.

Over recent years, there has been a keen uptake in the use of microperimetry (MP) in the research field. Notably, it is listed as a primary or secondary endpoint or keyword in over 150 clinical studies registered online with the US National Library of Medicine [17]. It has been used to help characterise a wide range of ocular conditions, including AMD [18], choroidal neovascularisation (CNV) [19], macular oedema arising from diabetes [20] or uveitis [21], central serous chorioretinopathy (CSCR) [22], retinal vein occlusion [23], birdshot chorioretinopathy [24], macular holes [25], epiretinal membranes [26], and IRD such as Stargardt disease [27], choroideremia [28-30], juvenile X-linked retinoschisis [31], and RPGRassociated X-linked retinitis pigmentosa [32]. To a lesser extent, MP has already been adopted as an endpoint in interventional retinal disease clinical trials. Given the continued interest in its potential, we sought to characterise this existing uptake, identifying current trends in its use and elucidating potential future directions in endpoint development and validation.

Following a technical review of commercially available microperimeters and their specifications, summarised in Table 1, this manuscript discusses MP as an endpoint in 
retinal disease clinical trials. Specifically, we review the use of retinal sensitivity measures as primary, secondary, or exploratory endpoints in interventional trials. To contextualise the review findings, we also discuss research with the potential to inform MP endpoint development such as natural history studies and studies discussing novel MP metrics. Though fixation stability metrics are provided by microperimeters, given the significant overrepresentation of retinal sensitivity in the literature and breadth of the subject matter, fixation is not explicitly addressed but will be briefly discussed for context.

\section{Introduction to MP}

MP is a psychophysical method which probes retinal sensitivity, specifically across the macula. MP is somewhat of a misnomer as the stimulus size (Goldmann size $\mathrm{I}-\mathrm{V}$ ) is comparable to that used in standard automated perimetry (SAP) and the retinal area covered is up to $30^{\circ}$ from the fovea [33]. Alternative terms considered more accurate include "fundus-guided perimetry" or "fundusrelated perimetry," but the original term has persisted since its first use in the literature in 1990, and thus MP will be used herein [34].

MP is distinguished from conventional visual fields by its ability to display and track a live fundus image during an exam, whilst adjusting for fixational eye movements. This provides assurance that threshold sensitivity values correspond to specific retinal locations. Diseases affecting the macula can result in unstable and/or eccentric fixation, making MP an attractive tool in their assessment [35]. Additionally, characteristics of fixation may change during disease progression and MP devices are able to quantify and track these changes.

The SLO-101 (Rodenstock, Munich, Germany) was the first commercially available device with MP capabilities. Somewhat rudimentary by today's standards, it lacked automation and repeat assessment of the same retinal locations was not easy to achieve. Furthermore, testing was arduous and the device was expensive to maintain. Thus, its use did not become widespread outside academic institutions and eventually it was taken off the market.

\section{Nidek Microperimeter-1}

The lack of automation was first addressed in 2002 with the arrival of the Nidek Microperimeter-1 (MP-1; Nidek Technologies, Padova, Italy), the first commercial- ly available MP device with an eye tracker. Using a baseline fundus image, high-contrast landmarks are manually selected and act as a reference, enabling eye tracking technology to detect changes in eye position every $40 \mathrm{~ms}$ (frequency $25 \mathrm{~Hz}$ ). Requiring a minimum pupil of approximately $4 \mathrm{~mm}$, pupil mydriasis is often required [36].

With a dynamic range of $0-20 \mathrm{~dB}$, stimuli are projected via a liquid crystal display (background luminance of $1.27 \mathrm{~cd} / \mathrm{m}^{2}$ ) according to either a standard (macular or peripapillary) or customised grid. Additional test points can be added to a default grid or created de novo anywhere across the central $40^{\circ}$ by way of an onboard "pattern editor." Fixation target characteristics are also customisable and stimuli can be presented in Goldmann sizes I-V in white or red.

A black and white infrared fundus image is viewable during testing. A high-quality colour image can be captured at the end of testing, onto which the sensitivity map can be superimposed. Retinal sensitivity maps can also be superimposed on imported images, including fundus autofluorescence (FAF), fluorescein, and indocyanine green angiography, thus allowing retinal sensitivity values to be directly associated with retinal lesions. Furthermore, it is possible to import a Heidelberg optical coherence tomography (OCT) line scan prior to testing, to help identify the anatomical fovea. This feature may be particularly desirable in patients with geographic atrophy (GA) where the fovea may otherwise not be readily identifiable.

According to the literature, the MP-1 has the largestscale normative database available for mesopic testing in a microperimeter, incorporating data from 190 healthy subjects between the ages of 20 and 75 years [37]. This allows generation of a "local defect map" which presents the differences between obtained sensitivity and agematched normal values, provided testing utilises Goldmann III stimuli within a $20^{\circ}$ diameter circle centred on the fovea. The MP-1 is also the only microperimeter to offer kinetic testing.

\section{Nidek MP-3}

Nidek released the MP-3 in 2014 with a larger dynamic range of $0-34 \mathrm{~dB}$ for light-adapted testing with the option of 2 background luminances: 1.27 or $10 \mathrm{~cd} / \mathrm{m}^{2}$. Later in 2018, the MP-3S was introduced, offering scotopic testing facilitated by a background luminance of 0.00095 $\mathrm{cd} / \mathrm{m}^{2}$ and a scotopic dynamic range of $0-24 \mathrm{~dB}$. MP-3S is the only microperimeter that can perform scotopic, mesopic, and photopic testing. 
Compared to the MP-1, MP-3 has improved eye tracking technology, tracking at $30 \mathrm{~Hz}$ and landmark identification is no longer required. Grid and fixation target customisation options have been retained and the same minimum pupil size and dilation recommendations apply. Having no normative database, the MP-3 cannot produce local defect maps and currently does not allow the import of OCT or other images. As kinetic testing was not widely adopted, this feature has been removed. If desired, the option to simulate MP-1 mesopic testing conditions and scale can be selected with the MP-3. This facilitates the continued longitudinal follow-up for patients who have had previous mesopic testing on MP-1. This is not possible for scotopic testing given the differences in scotopic background luminance.

\section{Optos OCT/SLO}

In 2006, the spectral OCT/SLO (OPKO/OTI, Miami, FL, USA), was launched, later renamed the Optos OCT/ SLO (Optos, Dunfermline, UK) when the technology was acquired by Optos in 2012. The OCT/SLO combines spectral-domain OCT (SD-OCT) with MP, thus allowing topographical alignment of retinal thickness and light sensitivity measurements, the only device to do so. En face confocal retinal images acquired by SLO (scanning laser ophthalmoscope) are simultaneously acquired alongside cross-sectional SD-OCT retinal images. Its dynamic range matches the MP- 1 and many testing parameters are customisable, including grid pattern, stimuli duration, shape, and colour. Though still in circulation, the OCT/SLO is no longer manufactured.

\section{MAIA}

The Macular Integrity Assessment (MAIA, CenterVue, Padova, Italy) microperimeter has been available since 2009. It utilises a near-infrared line confocal SLO for fundus imaging and a light-emitting diode stimulus projector focused on the retina. Instead of using reference landmarks, the MAIA eye tracker registers the entire fundus image, tracking each pixel at $25 \mathrm{~Hz}$. With a background luminance of $1.27 \mathrm{~cd} / \mathrm{m}^{2}$, it tests function in the mesopic range and has a dynamic range of $0-36 \mathrm{~dB}$, comparing favourably to other devices. A newer version of the device, the S-MAIA, is able to perform scotopic testing in addition (background luminance of $<0.0001$ $\left.\mathrm{cd} / \mathrm{m}^{2}\right)$.

Microperimetry: Trial Endpoint in Retinal Disease
One notable advantage of this device is it requires only a $2.5-\mathrm{mm}$ minimum pupil size, often negating the need for pupil dilation. The MAIA provides a bank of standard grids and fixation targets of circles and crosses. Though customised grids can be imported to the MAIA, they must be programmed in an XML (Extensible Markup Language) file and uploaded to the device.

An age-matched normative database for subjects aged 20-80 years old, compiled by the manufacturer, informed their development of a macular integrity index. This index is provided in the results under limited circumstances (i.e., for mesopic testing with a 4-2 staircase strategy and with a standard grid of $10^{\circ}$ diameter containing 37 radially oriented points centred on the fovea). The macular integrity index categorises the retinal sensitivity test results into 1 of 3 groups: normal, suspect, or abnormal. A numerical summary value is also provided, with larger values representing a higher likelihood that test findings are abnormal. This is not indicative of disease severity and is distinct from $\mathrm{dB}$ sensitivity values.

\section{Compass}

The Compass (CenterVue) was released in 2015. Tailored specifically for use in glaucoma, it shares the luminous parameters and sensitivity scales of SAP. With a $60^{\circ}$ field of view, it offers 10-2, 24-2, and 30-2 threshold testing, but customised grids are not available. A minimum $3-\mathrm{mm}$ pupil is required, obviating the need for pharmacological mydriasis.

Using confocal SLO technology for tracking, it is able to generate true colour confocal images as well as red-free images of the optic nerve head. Two threshold strategies are available: $4-2$ staircase and Zippy Estimation by Sequential Testing (ZEST), the latter being an established adaptive Bayesian algorithm that aims to shorten the testing time, like the Swedish Interactive Thresholding Algorithm (SITA) algorithms of SAP. Normative data are incorporated in its software, thus allowing typical SAP measures such as mean deviation and pattern standard deviation plus false negative and false positive reliability indices.

Common to all MP devices discussed so far, Compass results can be viewed as typical topographic retinal sensitivity maps (i.e., superiorly projected stimuli represented on superior retina), but results are also shown as conventional visual field maps, whereby stimuli are displayed according to their location in visual field space (i.e., superiorly projected stimuli represented in inferior visual field space).

Ophthalmologica 2021;244:418-450 
The influence of SAP on the design of MP devices is evident in the specifications for their light-adapted testing conditions which have been modelled on those used in popular perimeters. Analogous to the Octopus perimeters (Haag-Streit AG, Koeniz, Switzerland), the MP-1, MP-3, and MAIA have a background luminance of 1.27 $\mathrm{cd} / \mathrm{m}^{2}$ for mesopic testing. MP-3 and Optos OCT/SLO's background luminance of $10 \mathrm{~cd} / \mathrm{m}^{2}$ for photopic testing is the same as Humphrey perimeters (Carl Zeiss Meditec, Jena, Germany) [38]. The key features of all the commercially available devices discussed are summarised in Table 1 .

\section{Scotopic Testing in MP}

In light conditions, MP primarily assesses the function of cone photoreceptors (photopic) or a mixture of cone and rod function (mesopic), as determined by the luminance of the testing conditions. Impaired rod function is known to occur in a range of retinal conditions including AMD [39], retinal telangiectasia [40], CSCR [41], congenital stationary night blindness [42], and rod-cone dystrophy [43]. Affected patients find dimly lit and low contrast conditions challenging. Isolation of photoreceptor activity is warranted to determine the impact of interventions targeting a particular photoreceptor type. Rod activity may be assessed by scotopic electrophysiological tests [44], dark-adapted perimetry [45, 46], and indices of dark adaption such as the rod-intercept time [47]. Scotopic MP is a welcome addition to the range of clinical tests available. In the case of AMD, it has provided functional evidence of early impaired rod function, confirming what was previously hypothesised from histological analysis $[48,49]$.

The scotopic capability of the MP-1S was modelled on a prototype developed by Crossland et al. [50]. Scotopic spectral sensitivity is maximal for light of wavelength 498-505 nm, which is also the peak absorption wavelength of rhodopsin. By adding a 500-nm short pass filter and a 2.0-neutral density (ND) filter to the optical pathway of the MP-1, luminance levels were attenuated to those suitable for scotopic testing. A $500-\mathrm{nm}$ short-pass filter blocks wavelengths of light above $500 \mathrm{~nm}$. A $2.0 \mathrm{ND}$ filter reduces the intensity by a factor of 100 (i.e., $10^{2}$ ); however, when the two are combined the overall effect is attenuation by a factor of 500 . The standard MP-1S model comes with these filters.

A 1.0-ND filter has also been used by researchers with the MP-1S to attenuate stimuli to the desired level accord- ing to an individual's sensitivity values [51]. The purpose of this, as will be discussed later, is to minimise ceiling and/or floor effects so that the attained threshold values mostly fall within the 0 - to $20-\mathrm{dB}$ dynamic range. However, no correlation between sensitivity values obtained with different ND filters has been validated, thus precluding the direct comparison of results from patients tested with different ND filters [51].

In 2018, scotopic function for the MP-3 (i.e., MP-3S) was introduced with the filters required for scotopic testing in-built. The MP-3S uses a more selective bandpass filter with a peak transmission at $500 \mathrm{~nm}$. Background illumination has been reduced further to $0.00095 \mathrm{~cd} / \mathrm{m}^{2}$ and this device has a dynamic range of $0-24 \mathrm{~dB}$. As previously mentioned, this change in background illumination means that longitudinal scotopic testing in patients commenced in the MP-1S cannot cross over to the MP-3S.

In turn, Centervue released the S-MAIA, whose scotopic feature presents stimuli in 2 different wavelengths: cyan $(505 \mathrm{~nm})$ and red $(627 \mathrm{~nm})$ which help to further isolate photoreceptor activity. This has been validated in a normative study [52]. Each grid location is tested with the cyan stimulus and then red stimulus testing follows thereafter. The S-MAIA generates average threshold sensitivity values for both scotopic cyan and red testing separately, and also subtracts red values from cyan to give a value for "cyan-red difference."

The concept of using 2 wavelengths of stimuli was first established in modified perimeters to isolate photoreceptor function by exploiting the difference in spectral sensitivity of rods and cones [53, 54]. Generally, under fully dark-adapted conditions in healthy retina outside the rod-free zone, rods are more sensitive than cones at both wavelengths and, secondly, the sensitivity to cyan is much higher (around 2 log units) than for red stimuli [53]. The scotopic setting of the S-MAIA has been calibrated according to the CIE 1951 scotopic luminosity function or $\mathrm{V}^{\prime}(\lambda)$ such that the radiance of a sensitivity value for scotopic cyan stimuli is in effect $20 \mathrm{~dB}$ lower than that for red [54]. Therefore, a cyan-red difference of around $0 \mathrm{~dB}$ (in areas outside the rod-free zone and in the presence of normal sensitivity values for cyan and red), indicates normal rod function in the S-MAIA [52].

In retinal disease, one may need to exercise caution when interpreting the results of 2-wavelength stimuli testing as it cannot be assumed that the sensitivity values obtained are mediated by the same photoreceptor type as for normal eyes (e.g., sensitivity values obtained for cyan stimulus outside the rod free zone which ordinarily would be mediated by rods may instead be mediated by cones in 
the presence of rod impairment). Interpretation should involve evaluation of the location of the tested area (given the differing topographical densities of rods and cones according to eccentricity); the cyan and red sensitivity values as compared to normative values but also compared to each other and lastly any device limitations such as floor effects.

To expand on this, in the S-MAIA, isolated rod dysfunction (or where rod dysfunction is greater than cone dysfunction) would be reflected in a reduction of cyan sensitivity, while red sensitivity would not be so affected, thus leading to a more negative value for cyan-red difference [55]. However, severe rod dysfunction, whereby cones mediate sensitivity to both cyan and red stimuli, may not be readily observed due to the floor effects of the device [55]. Isolated cone dysfunction may lead to reduction in scotopic red sensitivity, especially at central retinal locations where the sensitivity values would have been expected to be maximal in a normal eye. Cyan sensitivity would not be as affected.

The first-generation S-MAIA had a dynamic range of 0-20 dB; however, extended minimum and maximum stimulus intensities were introduced in the second-generation device providing an extended dynamic range of 0-36 dB. Via software upgrade, first-generation data could be automatically converted to equivalent secondgeneration values. However, points $<0 \mathrm{~dB}$ on the firstgeneration tests are converted to $<10 \mathrm{~dB}$ as they cannot be further quantified. These changes were accompanied by a change in staircase strategy from $2-1$ to $4-2$, thus a direct comparison of first- and second-generation SMAIA data is not, in the very strictest sense, feasible.

\section{Dark Adaptation}

To probe scotopic rod-mediated function, a period of dark adaptation (DA) is required prior to testing. The period is based on our understanding of the DA curve, which plots retinal sensitivity over time when a transition is made from light to dark conditions following a period of bleaching or bright light exposure. The initial rapid increase in sensitivity is mediated by cone photoreceptors and after several minutes reaches a plateau referred to as the cone-rod break. Beyond this period, rods, which are much more operative under scotopic conditions, mediate the further increase in sensitivity, reaching their maximum sensitivity after around 30-40 min of DA [56]. DA periods for scotopic MP are typically cited as $30 \mathrm{~min}$ [50, $55,57-59]$ although slightly longer DA periods have also been used [60]. Given the additional burden to the patient and the impact on overall examination time, the duration of DA should consider both practical and physiological constraints.

Based on S-MAIA data from normal controls and patients with choroideremia, if starting from normal ambient light conditions, no period of DA is required for mesopic testing provided the eye has relatively preserved cone function [61]. A 10 -min period of DA is recommended if an eye has had recent exposure to bright light (such as retinal photography or slit lamp biomicroscopy). Therefore, the schedule of tests prior to an MP test should be considered.

\section{Scales Used in MP}

The scale used in MP follows a similar convention to SAP. To account for the wide range of luminance an eye is responsive to, a logarithmic scale with decibel units is used to measure retinal sensitivity. One decibel unit corresponds to a $0.1-\log$ unit change. For example, a dynamic range of $0-20 \mathrm{~dB}$ relates to the differential luminance at the maximum stimulus intensity being $2 \mathrm{log}$ units $\left(10^{2}=100\right.$ times $)$ greater than that at the minimum stimulus intensity. Poorly sensitive areas of retina require brighter stimulus intensities to reach threshold detection. However, as it is intuitive to have low decibel values representing poorer retinal sensitivities, an inverse logarithmic scale is adopted.

Importantly, the decibel range is not an absolute scale and thus the same value in decibels is not the same from one device to another. As such, longitudinal analysis should be performed using the same microperimeter, facilitated by follow-up mode which allows repeat automated testing of the same retinal points regardless of baseline fixation or its subsequent change over time.

The scale for a given device is fixed according to the maximum stimulus intensity available, that is $0 \mathrm{~dB}$, representing the lowest retinal sensitivity that is quantifiable (i.e., correct response to the brightest stimulus intensity is registered). Floor effects refer to the occasions where the observer was not able to detect the brightest stimulus, and therefore the depth of defect cannot be further quantified. These are nominally assigned $<0 \mathrm{~dB}$ or $-1 \mathrm{~dB}$ values. Thus, floor effects, by their nature, may result in underestimation of the defect and represent a heterogeneous group of sensitivity losses.

In determining the impact of an intervention, trialists need to consider where in the dynamic range of the mea- 
surement tool the values derived from the patient population fall. For phase I/II trials establishing safety, patients with severe disease are often recruited. If at preintervention testing patients encounter significant floor effects, the opportunity to track meaningful change post-intervention is reduced, and both deterioration and improvement may be masked. For example, patients with neovascular AMD encountered floor effects under mesopic, scotopic cyan, and scotopic red testing despite the 0-36 $\mathrm{dB}$ dynamic range of the S-MAIA [58]. Thus, the authors propose patients with mild to moderate disease may make better candidates for interventional studies.

This, however, does not mean the occurrence of floor effects precludes the ability to track meaningful change completely. Although of limited, strictly quantifiable use, the proportion of points reaching the floor can be tallied up and compared over time or pre- versus postintervention. Additionally, scotomas are, by definition, areas of diminished vision surrounded by normal or relatively preserved vision. Therefore, they will commonly, and unavoidably, consist of values which approach or reach the floor. In fact, research groups have defined scotoma-related outcome measures according to type (absolute or relative) and size. That said, static testing may be inferior to kinetic testing when assessing the size and borders of scotoma, as the latter technique is not constrained by set spacing intervals between points of a grid [62].

\section{Comparisons across MP Devices}

Despite the differences in testing conditions and strategies of MP devices, numerous studies have compared their functions by performing testing using different microperimeters on the same subjects. In both SAP and $\mathrm{MP}$, the task required of the subject is to distinguish the stimulus from its surrounding background. Where devices employ the same background luminance, their decibel scales can theoretically be aligned to each other by considering each unit of the respective scale in terms of their differential luminance value (calculated as background luminance subtracted from luminance at the site of stimulus projection, described in detail by Parodi et al. [63] as well as Vujosevic et al. [64]). For example, a differential luminance of approximately $127 \mathrm{~cd} / \mathrm{m}^{2}$ corresponds to 4 and $0 \mathrm{~dB}$ on the MAIA and MP-1 mesopic decibel scales, respectively. Thus, by adding $4 \mathrm{~dB}$ to the MP-1 sensitivity value, one should arrive at the corre- sponding value in MAIA. In practice, this 4-dB difference is often not observed, with substantial deviations evident $[63,65]$. For example, patients with IRD with an average mean sensitivity (MS) of $5.68 \mathrm{~dB}$ on MP-1 were found to have an average MS of $14.66 \mathrm{~dB}$ in MAIA (instead of the expected $9.66 \mathrm{~dB}$ ) [63]. In the same study, normally sighted subjects with an average mesopic MS of $18.46 \mathrm{~dB}$ on MP-1 had an observed average MAIA mesopic MS of $28.52 \mathrm{~dB}$ (rather than $22.44 \mathrm{~dB}$ ). In pointwise sensitivity (PWS), an average difference of $+7.3 \mathrm{~dB}$ was found when comparing mesopic MP-1 to MAIA values in a mixed population group (normally sighted subjects and those with visual impairment) [65]. There was a 95\% limit of agreement of -3.9 to $18.5 \mathrm{~dB}$, considered too wide-ranging to be of much clinical use. Similarly, in patients with $A M D$, a pointwise difference ranging between -14 and $6 \mathrm{~dB}$ was seen in mesopic MAIA and MP- 1 testing (although the median correction was MAIA = MP-1 $+2 \mathrm{~dB})[66]$.

For device comparisons where the background luminances are not the same, Weber contrast (the differential luminance divided by the background luminance for that device) can be calculated for each unit of the respective device's scale. The decibel scales of the 2 devices are thus matched by the common scale of contrast values (i.e., the contrast value relating to a specified decibel value for 1 device is aligned to the same contrast value in the other device's decibel scale as explained, e.g., by Liu et al. [38]). Using this method, no difference in average thresholds, expressed as contrast values, was found between MAIA and Optos OCT/SLO in normal subjects [67]. This was not the case for the visually impaired patients in the same study and it was postulated that the brighter stimuli required may increase variability due to increased stray light effects. The OCT/SLO has also been compared with MP-1 via contrast values but found to correlate poorly [38]. It would be important to note that although one can theoretically align devices' scales according to contrast, different, not directly comparable physiological systems may be at work (e.g., mesopic with MP-1 and photopic with OCT/SLO).

However, MAIA and MP-3 utilise similar testing conditions but still generate differing mesopic retinal sensitivity values for normal subjects. Adding a "correction factor" of $5.65 \mathrm{~dB}$ to the MP-3 value to obtain the MAIA value allowed a strong statistically significant correlation to be demonstrated [68]. Possible explanations given for this disparity included differences in the systems used for stimulus projection and for grid placement onto the fundal image. 


\section{Reliability Indices}

It is imperative that any clinical trial measurements are reliable and, like SAP, MP offers indices against which the reliability of a test result can be gauged. However, in MP these indices are less evolved and not consistently available or applied.

In SAP, reliability indices refer to false positives, false negatives, and fixation losses, the assessment of which classically requires the presentation of additional tests, socalled "catch trials," typically making up 3-5\% of stimuli presentations [69]. False positives refer to instances where a response is recorded when no stimulus is presented. These are either responses made when a stimulus is not presented but is anticipated to be, according to the expected "rhythm" of stimuli presentation. Alternatively, the response time following stimuli presentation can be analysed. The minimum response time to react to a stimulus is known to be around $180 \mathrm{~ms}$ [70]. Adjusted for a subject's mean response time, this period defines "response windows" (when a response is expected to occur) and "listening windows" (when a response is not expected). Responses occurring in the "listening window" are considered to be a false positive [71]. False negative catch trials involve the presentation of suprathreshold stimuli at locations in which the threshold has already been determined. Fixation losses are characterised according to the Heijl-Krakau method which involves assessing the subject's responses to stimuli presented at the optic nerve head [72].

In MP, the situation is more fragmented. For instance, the MP-1S measures false positives by presenting stimuli at the optic nerve head, whereas the MP-3 characterises a false positive as a response made in the absence of a stimulus. The S-MAIA does not assess false positives or false negatives, but does provide an index referred to as "fixation losses." However, these fixation losses are also assessed using optic nerve head stimuli presentations (with a $10-\mathrm{dB}$ intensity stimuli presented every $60 \mathrm{~s}$ when testing under full threshold, 4-2 strategy conditions). In fact, in the literature, researchers using MAIA often refer to this fixation loss metric as a false positive rate $[58,60,73]$. Generally, false negatives are not provided in MP devices but are available on the MP-3. A specific consideration for scotopic testing is that repeat testing and the presentation of suprathreshold stimuli may have the potential to disturb scotopic conditions.

It could also be argued that "fixation losses" are not as relevant for MP as for SAP given that MP detects and compensates for retinal movements directly. This likely explains why researchers have moved away from this term, preferring the term "false positive" instead. Furthermore, accurate marking of the optic nerve head centre is essential if fixation losses/false positives are to be accurately represented. This is because any off-centre misplacement, especially in subjects with small optic discs, may render the stimulus visible due to stray light.

The S-MAIA manual states that fixation losses over $30 \%$ are unreliable. Published reports differ according to the level of fixation losses deemed tolerable, with research groups defining their own cut-offs, such as 15, 25, and $33 \%$ [74-76]. Given the small number of catch trials presented, 1 or 2 accidental button presses may be enough to classify an examination as unreliable. Available in later S-MAIA software versions, some groups have analysed "wrong pressure event" raw data as a surrogate for the false positive rate, calculated as the number of wrong pressure events divided by the test duration $[58,60]$. A wrong pressure event is a response occurring $1,500 \mathrm{~ms}$ or more after a stimulus presentation and prior to the next stimulus presentation.

The relative contributions of numerous S-MAIA reliability indices to variance in between-subject PWS test-retest variability (TRTV) have been statistically explored in both neovascular AMD and GA $[58,60]$. The parameters analysed included: false positive (blind spot presentation), wrong pressure event rate, examination duration time, and fixation stability (95\% bivariate contour ellipse area; BCEA). In neovascular AMD, false positives were the most important factor for mesopic and scotopic red testing, whilst a wrong pressure event rate had the greatest impact for scotopic cyan testing [58]. In those with GA, mean retinal sensitivity was the largest determinant of the variance of mesopic and scotopic cyan/red testing and a wrong pressure event rate was more informative than false positives. This suggests indices other than a false positive rate (termed "fixation loss" by device) should also be considered when establishing inclusion criteria for test reliability in trial protocols. Such criteria may differ according to type of testing (mesopic, scotopic cyan/red) and pathology.

\section{MP Retinal Sensitivity Indices and Analysis}

The native software of microperimeters provide a limited range of retinal sensitivity indices. The most widely reported of these is MS: the arithmetic average sensitivity across all grid locations. The display of results also pres- 
Table 2. MP retinal sensitivity metrics provided by device compared to researcher-derived metrics in the literature

\begin{tabular}{ll}
\hline Endpoint metrics provided by device & Researcher-derived metrics from raw data \\
\hline Mean sensitivity & Mean sensitivity of subsections of grid \\
- Mean of all points in a test grid & - Based in eccentricity from fovea, that is CMS or PMS \\
Pointwise sensitivity & - Of non-scotomatous points (defined at baseline) \\
- Individual sensitivity at each point & Change in PWS \\
Interpolated colour "heat maps" & - Change to PWS over time \\
- PWS values expressed according to a colour gradient, & - Change in number of points reaching a certain threshold sensitivity \\
superimposed on a fundus image & value \\
& Scotoma size defined by number or \% of \\
& - Absolute scotoma points \\
& - Relative scotoma points \\
& Number of "seeing" versus "non-seeing" points \\
& Volumetric indices derived using hill-of-vision modelling software \\
\hline
\end{tabular}

PWS, pointwise sensitivity; CMS, central mean sensitivity; PMS, paracentral mean sensitivity.

${ }^{1}$ Cut-offs for relative and absolute vary by study.

Table 3. Examples of MP features and raw data used to define condition- or treatment-specific metrics

\begin{tabular}{ll}
\hline Condition & Customisation of MP settings \\
\hline Choroideremia & Grid customised according to intact retina identified by FAF \\
$\begin{array}{l}\text { Targeted treated areas (e.g., gene therapy vector } \\
\text { bleb; area of stem cell graft) }\end{array}$ & $\begin{array}{l}\text { Grid customised to demarcate treated versus untreated areas for direct compari- } \\
\text { son }\end{array}$ \\
\hline Stargardt disease & MS of edge of scotoma points \\
\hline Early/intermediate AMD & $\begin{array}{l}\text { PWS over specific retinal lesion identified on OCT (i.e., reticular pseudodrusen, } \\
\text { large drusen, nascent GA) versus unremarkable regions } \\
\text { Percent reduced threshold (\% of points with abnormal retinal sensitivity defined } \\
\text { as <25 dB on MAIA) }\end{array}$ \\
\hline Geographic atrophy & $\begin{array}{l}\text { MS of peri-lesional points (points immediately adjoining a point where brightest } \\
\text { stimuli unseen) } \\
\text { Deep scotoma mapping strategy }\end{array}$ \\
\hline Macular telangiectasia & Aggregate sensitivity loss \\
\hline Diabetic macular oedema & MS over OCT subfields \\
\hline
\end{tabular}

AMD, age-related macular degeneration; FAF, fundus autofluorescence; GA, geographic atrophy; OCT, optical coherence tomography; PWS, pointwise sensitivity.

ents individual sensitivity values for each grid location (PWS) both numerically and visually, according to a colour gradient. Given this limited range, research groups have maximised the use of raw retinal sensitivity data, devising alternative metrics of interest which feature heavily in interventional retinal disease trials, as will become apparent shortly. These broadly fall into 2 catego- ries: subdivisions of MS and PWS, and scotoma evaluation. For quick reference, Table 2 summarises both device and researcher-derived metrics. In addition, and as presented in Table 3, condition- or treatment-specific characteristics and outcomes have also been conceived by researchers, and these will be discussed in relation to their pathology. 
Table 4. Examples of $95 \%$ CoR for a variety of retinal diseases reported in the literature

\begin{tabular}{lllll}
\hline Condition & Device & Test & PWS CoR & MS CoR \\
\hline $\begin{array}{l}\text { Mixed macular diseases } \\
\text { Chen et al. [78], 2009 }\end{array}$ & MP-1 & Mesopic & $\begin{array}{l}5.56 \mathrm{~dB}(4.95 \mathrm{~dB} \text { if floor/ } \\
\text { ceiling effects removed) }\end{array}$ & $1.81 \mathrm{~dB}$ \\
\hline $\begin{array}{l}\text { Stargardt disease } \\
\text { Cideciyan et al. [190], 2012 }\end{array}$ & $\begin{array}{l}\text { MP-1 (custom grid; } \\
\text { red stimulus) }\end{array}$ & Mesopic & $4.2 \mathrm{~dB}$ & N/A \\
\hline $\begin{array}{llll}\text { XLRS } \\
\text { Jeffrey et al. [188], 2014 }\end{array}$ & MP-1 & Mesopic & $6.8 \mathrm{~dB}$ (better eye) & $2.2 \mathrm{~dB}$ (better eye) \\
& & & $\begin{array}{l}5.4 \mathrm{~dB} \text { (worse eye) } \\
\text { Floor/ceiling effects removed }\end{array}$ & Floor/ceiling effects removed \\
\hline $\begin{array}{l}\text { Macular telangiectasia } \\
\text { Wong et al. [99], 2017 }\end{array}$ & MAIA & Mesopic & $7.20 \mathrm{~dB}$ & $2.60 \mathrm{~dB}$ \\
\hline $\begin{array}{l}\text { Intermediate AMD } \\
\text { Welker et al. [59], 2018 }\end{array}$ & S-MAIA & $\begin{array}{l}\text { Mesopic } \\
\text { Scotopic }\end{array}$ & $4.40 \mathrm{~dB}$ & N/A dB \\
\hline $\begin{array}{l}\text { RPGR retinitis pigmentosa } \\
\text { Buckley et al. [189], 2020 }\end{array}$ & MAIA & Mesopic & $6 \mathrm{~dB}$ & $1.30 \mathrm{~dB}$
\end{tabular}

PWS, pointwise sensitivity; CoR, 95\% co-efficient of repeatability; MS, mean sensitivity; AMD, age-related macular degeneration; XLRS, X-linked retinoschisis; N/A, not applicable/available.

\section{Mean and Pointwise Sensitivity}

Taken at face value, MS is arguably a simple measure; however, further reflection is warranted. As a global outcome, MS runs the risk of missing localised pathological variation in sensitivity $[74,77]$ as the difference in sensitivity across grid points is reduced by virtue of averaging. To retain some topographical information, MS may be calculated for subsections of a grid. An example of this is the categorisation of a grid into central and paracentral areas, with MS calculated for each separately (CMS and PMS), as per Chen et al. [78]. Derivations of CMS and PMS, varying by underlying grid design and expanse of central and paracentral areas, are commonly encountered in the literature [79-87].

The constituent PWS should be examined to identify floor or ceiling effects, as in their presence they can cause the resultant MS to be over- or underestimated, potentially masking true change in MS across the tested region over time. Attempts have been made to account for floor effects by tracking the MS of only those points with a measurable threshold (i.e., non-absolute scotoma points) [88-90]. Conversely, the approach of stratifying participants by baseline MS value has been used to mitigate ceiling effects, whereby changes in MS are separately examined in those whose MS is reduced at baseline [81]. To account for considerable variability in observed pointwise measures (i.e., scotomatous and non-scotomatous regions), Hood et al. [91] proposed calculating MS on a linear scale. Conceptualised using SAP data in glaucoma, this method involves averaging anti-logged individual pointwise values before taking the log again.

MS is also inextricably influenced by the grid design. The total number of points, their spacing and their configuration will impact the information obtained. Commonly, grids have more central than peripheral points, weighting MS in favour of foveal sensitivity. The use of different grids across studies also hinders the direct comparison of MS. One method of addressing this is hill-of-vision volumetric analysis such as that performed by Visual Field Modelling and Analysis (VFMA) software. Within the boundaries of a test grid, the operator can select a circular retinal area. The threshold sensitivities within this area are modelled to generate an interpolated volume sensitivity index, expressed in units of decibel-steradians (dB-sr), with higher values equating to better sensitivity. Although originally used with perimetry data, its use with MP raw data has been described more recently, including for Stargardt disease [27], achromatopsia [92, 93], and to evaluate the area of transplanted retinal pigment epithelium graft [94].

As with any measure, MS and PMS are subject to measurement error and variability. To be better equipped to distinguish disease progression from such variability, 
TRTV should be determined, ideally specific to the disease and device.

TRTV is conventionally defined by the $95 \%$ BlandAltman co-efficient of repeatability (CoR) and is interpreted as a value for which $95 \%$ of test-retest differences for a subject are expected to fall, with smaller values indicating lower variability [95]. TRTV can be calculated for both PWS and MS. Understandably, PWS CoR is higher than that of MS, as it does not profit from the averaging effects of the latter. As such there is a trade-off between the precision of pointwise measures and repeatability. Table 4 , although not exhaustive, is provided to familiarise the reader with the ranges of CoR that have been reported for various retinal conditions using different MP devices.

\section{Scotoma}

Using raw MP data, simplistic scotoma-driven outcomes have also been specified by research groups, such as the percentage or number of reduced, relative, or absolute scotomatous points [96-98]. Cut-offs for what is considered reduced, relative, and absolute loss vary across studies. Due to the customisable nature of MP testing grids, including differing stimuli counts and spacing used, care is advised in the interpretation of such metrics across trials. Repeatability of such measures has been described using a 37-stimuli grid in macula telangiectasia [99]. A CoR of 5 was found for absolute scotomatous points and 13 for normal sensitivity points $(>25 \mathrm{~dB}$ on MAIA). With variability representing 35\% of the total scale in this case, the evaluation of the number of normal sensitivity points may be of limited use.

\section{Fixation Stability}

Microperimeters assess fixation stability throughout MP examinations or as standalone assessment. Fixation attempts are mapped onto the fundus image as a "cloud" of points indicating the position and stability of fixation. The location of the "cloud" reveals the retinal area used for fixation, the preferred retinal locus (PRL). The stability of fixation relates to the size of this area. Fuji et al. [100] described a method of quantifying fixation stability based on the percentage of fixation points within 2 and $4^{\circ}$ diameter circles centred on the gravitational centre of all fixation points. Fixation is categorised as "stable" if more than $75 \%$ of fixation points fall within a $2^{\circ}$ circle, "relatively unstable" if fewer than $75 \%$ fall within a $2^{\circ}$ circle but more than $75 \%$ fall within a $4^{\circ}$ circle, and "unstable" if fewer than $75 \%$ fall within a $4^{\circ}$ circle.

First described by Steinman [101], and reintroduced by Crossland et al. [102], the BCEA has become a more prominently used measure to characterise fixation. BCEA is the area in minutes of $\operatorname{arc}^{2}$ encompassing a defined percentage of fixation points, where higher values denote worse fixation. BCEA correlates more closely with reading speed and BCVA than the Fujii classifications [102, 103]. Although outside the scope of this review, fixation location and stability are also being investigated as potential endpoint measures, most notably in Stargardt disease [104-106].

\section{Review of MP Retinal Sensitivity Endpoints in Interventional Trials}

In order to evaluate the adoption of MP retinal sensitivity as an endpoint in clinical trials to date, we conducted a literature review in Embase and Ovid Medline during September 2020. The results of 2 main searches were combined. First, MP free-text search terms were combined with the Boolean operator OR (microperimet*, fundus controlled perimet*, fundus-controlled perimet*, fundus automated perimet*, fundus-automated perimet*, retinal sensitivit*, macular sensitivity*). Perimetry was also included as an MeSH term. Second, endpoint free-text search terms were used combined with OR (endpoint*, outcome measure*). A clinical trial MeSH term was also included. The 2 search results were combined with AND. Only articles in English were considered and conference articles or those using fixation stability-only endpoints were excluded.

Studies were further categorised according to whether subjects had acquired retinal disease or IRD. Given the immense phenotypic variety in retinal disease and the impact this has on qualities such a repeatability and trial design, we considered inherited and acquired disease separately. Abstracts referring to acquired disease were reviewed to identify those describing randomised interventional studies stating MP retinal sensitivity as a primary or secondary endpoint. As randomised trials are not commonplace in IRD, all interventional IRD studies employing MP were retained. Tables 5 and 6 provide a summary of all articles reviewed in the IRD and acquired categories, respectively, highlighting the eye condition, study design, intervention, MP test parameters employed, and endpoints utilised. 
Table 5. Summary of interventional studies in inherited retinal disease and their endpoints

\begin{tabular}{|c|c|c|c|c|}
\hline Study & Study design and interventions & Condition & Microperimetry test parameters & Endpoints \\
\hline $\begin{array}{l}\text { MacLaren et al. [107], } 2014 \\
\text { NCT01461213 }\end{array}$ & $\begin{array}{l}\text { Phase I/II } \\
\text { Subretinal AAV2.REP1 }\end{array}$ & Choroideremia & $\begin{array}{l}\text { MAIA } \\
\text { Mesopic } \\
20 \text { min DA } \\
\text { Custom grid tailored to intact macular areas } \\
\text { identified on FAF }\end{array}$ & $\begin{array}{l}\text { Primary } \\
\text { BCVA } \\
\text { Secondary } \\
\text { MP: } \\
\text { - Change in maximal point sensitivity } \\
\text { - Changes in MS } \\
\text { - Dimmest stimulus seen } \\
\text { - Total No. of points seen } \\
\text { OCT thickness } \\
\text { FAF area }\end{array}$ \\
\hline $\begin{array}{l}\text { Xue et al. [28], } 2018 \\
\text { NCT01461213 } \\
\text { (final outcome of MacLaren } \\
\text { et al. [107]) }\end{array}$ & $\begin{array}{l}\text { Phase I/II } \\
\text { Subretinal AAV2.REP1 }\end{array}$ & Choroideremia & $\begin{array}{l}\text { MAIA } \\
\text { Mesopic } \\
20 \text {-min DA } \\
\text { Followed protocol in MacLaren et al. [107] but } \\
\text { also } 10 \text { and } 20^{\circ} \text { grids used in some pts, according } \\
\text { to floor effects encountered }\end{array}$ & $\begin{array}{l}\text { Primary } \\
\text { BCVA } \\
\text { Secondary } \\
\text { MS } \\
\text { OCT-retinal thickness } \\
\text { FAF area }\end{array}$ \\
\hline $\begin{array}{l}\text { Dimopoulos et al. [29], } 2018 \\
\text { NCT02077361 }\end{array}$ & $\begin{array}{l}\text { Phase I } \\
\text { Subretinal AAV2.REP1 }\end{array}$ & Choroideremia & $\begin{array}{l}\text { MAIA } \\
\text { Mesopic } \\
20 \text {-min DA } \\
\text { Standard grid of } 37 \text { points for } 5 \text { pts } \\
10-2 \text { grid of } 61 \text { points for } 1 \mathrm{pt}\end{array}$ & $\begin{array}{l}\text { Primary } \\
\text { Safety (AEs, and assessed by OCT, FAF) } \\
\text { Secondary } \\
\text { BCVA } \\
\text { MS } \\
\text { Areas of intact RPE on FAF }\end{array}$ \\
\hline $\begin{array}{l}\text { Lam et al. [30], } 2019 \\
\text { NCT02553135 }\end{array}$ & $\begin{array}{l}\text { Phase II } \\
\text { Subretinal AAV2.REP1 }\end{array}$ & Choroideremia & $\begin{array}{l}\text { MAIA } \\
\text { Mesopic } \\
\text { Grid not specified }\end{array}$ & $\begin{array}{l}\text { Primary } \\
\text { BCVA, AEs } \\
\text { Secondary } \\
\text { MS } \\
\text { FAF area } \\
\text { OCT parameters }\end{array}$ \\
\hline $\begin{array}{l}\text { Fischer et al. [205], } 2019 \\
\text { NCT02671539 } \\
\text { (24-month data of Fischer } \\
\text { et al. [109]) }\end{array}$ & $\begin{array}{l}\text { Phase II } \\
\text { Randomisation of eye } \\
\text { Subretinal AAV2.REP1 }\end{array}$ & Choroideremia & $\begin{array}{l}\text { MAIA } \\
\text { Mesopic } \\
30 \text {-min DA } \\
10-2 \text { grid with } 68 \text { points }\end{array}$ & $\begin{array}{l}\text { Primary } \\
\text { BCVA } \\
\text { Secondary } \\
\text { MS } \\
\text { FAF changes } \\
\text { OCT parameters }\end{array}$ \\
\hline $\begin{array}{l}\text { Fischer et al. [109], } 2020 \\
\text { NCT02671539 } \\
\text { (12-month data with } \\
\text { focus on retinal sensitivity) }\end{array}$ & $\begin{array}{l}\text { Phase II } \\
\text { Randomisation of eye } \\
\text { Subretinal AAV2.REP1 }\end{array}$ & Choroideremia & $\begin{array}{l}\text { MAIA } \\
\text { Mesopic } \\
10-2 \text { grid with } 68 \text { points } \\
\text { (if }<6 \text { points seen on above grid, a } 37 \text {-point, } 10^{\circ} \\
\text { coverage grid was used) }\end{array}$ & $\begin{array}{l}\text { Primary } \\
\text { BCVA, safety } \\
\text { Secondary } \\
\text { MS } \\
\text { Maximal point retinal sensitivity } \\
\text { FAF changes } \\
\text { OCT parameters }\end{array}$ \\
\hline $\begin{array}{l}\text { Bainbridge et al. [111], } 2008 \\
\text { Bainbridge et al. [110], } 2015 \\
\text { (Final outcome of [111]) } \\
\text { NCT00643747 }\end{array}$ & $\begin{array}{l}\text { Phase I/II } \\
\text { Subretinal AAV2/2.hRPE65p. } \\
\text { hRPE65 }\end{array}$ & $\begin{array}{l}\text { Leber congenital } \\
\text { amaurosis }\end{array}$ & $\begin{array}{l}\text { MP-1 } \\
\text { Mesopic } \\
\text { 10-min DA } \\
55 \text {-point grid, appears to be positioned over } \\
\text { superotemporal arcade (site of retinotomy) [111] } \\
2 \text { types of grids used in all pts: } \\
\text { Central ( } 68 \text { pts) and peripheral [110] } \\
\text { Goldmann V } \\
4-2 \text { staircase }\end{array}$ & $\begin{array}{l}\text { Primary } \\
\text { Inflammation, AEs } \\
\text { Secondary } \\
\text { Visual function } \\
\text { [BCVA, kinetic perimetry, MP (pointwise } \\
\text { sensitivity), DA perimetry, mobility, CS, } \\
\text { colour vision, spectral sensitivity, retinal } \\
\text { imaging ERG] }\end{array}$ \\
\hline $\begin{array}{l}\text { Le Meur et al. [118], } 2018 \\
\text { NCT01496040 }\end{array}$ & $\begin{array}{l}\text { Phase I/II } \\
\text { Subretinal AAV2 or AAV4 } \\
\text { RPE65-RPE65 }\end{array}$ & $\begin{array}{l}\text { Leber congenital } \\
\text { amaurosis }\end{array}$ & $\begin{array}{l}\text { MP-1 } \\
\text { Mesopic } \\
\text { 10-min DA } \\
\text { Grid not specified }\end{array}$ & $\begin{array}{l}\text { Primary } \\
\text { AEs, biodistribution of viral vectors (in } \\
\text { urine, nasal samples, blood) } \\
\text { Secondary } \\
\text { ERG, BCVA, pupillometry, MP - MS; No. } \\
\text { of microscotomas } \\
\text { Mobility }\end{array}$ \\
\hline $\begin{array}{l}\text { Cukras et al. [31], } 2018 \\
\text { NCT02317887 }\end{array}$ & $\begin{array}{l}\text { Phase I/II } \\
\text { Intravitreal AAV8-RS1 }\end{array}$ & XLRS & $\begin{array}{l}\text { MP-1 } \\
\text { Mesopic } \\
68 \text { points } \\
10-2 \text { grid }\end{array}$ & $\begin{array}{l}\text { Primary } \\
\text { AEs, inflammation } \\
\text { Secondary } \\
\text { Visual function, ERG, AAV antibodies, } \\
\text { OCT changes } \\
\text { MP - No. of points which did not reach } \\
\text { floor vs. floor effects (dense scotomatous } \\
\text { points); } \\
\text { MS of responding points } \\
\text { MS of extra-scotomatous points } \\
\text { MS of para-scotomatous points }\end{array}$ \\
\hline
\end{tabular}


Table 5 (continued)

\begin{tabular}{|c|c|c|c|c|}
\hline Study & Study design and interventions & Condition & Microperimetry test parameters & Endpoints \\
\hline $\begin{array}{l}\text { Cehajic-Kapetanovic } \\
\text { et al. [32], } 2020 \\
\text { NCT03116113 }\end{array}$ & $\begin{array}{l}\text { Phase I/II } \\
\text { Subretinal AAV8-codon opti- } \\
\text { mised RPGR }\end{array}$ & RPGR RP & $\begin{array}{l}\text { MAIA } \\
\text { Mesopic } \\
68 \text { points } \\
10-2 \text { grid }\end{array}$ & $\begin{array}{l}\text { Primary } \\
\text { Safety } \\
\text { Secondary } \\
\text { BCVA, MS, central retinal thickness }\end{array}$ \\
\hline $\begin{array}{l}\text { Fischer et al. [126], } 2020 \\
\text { NCT02610582 }\end{array}$ & $\begin{array}{l}\text { Phase I/II } \\
\text { Subretinal AAV8.CNGA3 }\end{array}$ & $\begin{array}{l}\text { CNGA3 } \\
\text { Achromatopsia }\end{array}$ & $\begin{array}{l}\text { MP-1 } \\
\text { Mesopic } \\
20^{\circ} \text { grid }\end{array}$ & $\begin{array}{l}\text { Primary } \\
\text { Safety, inflammation } \\
\text { Secondary } \\
\text { Change in visual function (BCVA, MS, } 2 \\
\text { and } 4^{\circ} \text { fixation stability, spatial and } \\
\text { temporal resolution, chromatic tests, } \\
\text { flicker fusion frequency, CS, pupillary } \\
\text { responses, FST, QoL) }\end{array}$ \\
\hline Lenassi et al. [206], 2013 & $\begin{array}{l}\text { Prospective, interventional } \\
\text { case series } \\
\text { Argon green laser to RPE } \\
\text { anterior to drusen }\end{array}$ & $\begin{array}{l}\text { Autosomal } \\
\text { dominant drusen } \\
\text { (EFEMP1-related } \\
\text { maculopathy) }\end{array}$ & $\begin{array}{l}\text { MP-1 } \\
\text { Mesopic } \\
\text { Humphrey } 10 \text { program }\left(76 \text { points, central } 20^{\circ}\right) \\
4-2 \text { staircase }\end{array}$ & $\begin{array}{l}\text { Primary } \\
\text { BCVA } \\
\text { Changes in PWS } \\
\text { Scotoma size (No. of points }<0 \mathrm{~dB} \text { ) } \\
\text { Drusen volume on OCT } \\
\text { Secondary } \\
\text { Safety, development of CNV }\end{array}$ \\
\hline $\begin{array}{l}\text { Mehat et al. [94], } 2018 \\
\text { NCT01469832 }\end{array}$ & $\begin{array}{l}\text { Phase I/II } \\
\text { Subretinal transplantation of } \\
\text { hESC-derived RPE }\end{array}$ & STGD1 & $\begin{array}{l}\text { MP-1 } \\
\text { Mesopic } \\
\text { Central } 20^{\circ} \text { (including coverage over transplanted } \\
\text { area) } \\
\text { Also a high-density grid to analyse transplanted } \\
\text { area }\end{array}$ & $\begin{array}{l}\text { Primary } \\
\text { Safety, tolerance } \\
\text { Secondary } \\
\text { Retinal structure and function by MP } \\
\text { (PWS, Hill of vision modelling using } \\
\text { VFMA) } \\
\text { OCT, perimetry (static and kinetic), } \\
\text { mERG }\end{array}$ \\
\hline $\begin{array}{l}\text { Yamamoto et al. [207], } 2012 \\
\text { UMIN-CTR clinical trials No. } \\
\text { JapicCTI-090748 }\end{array}$ & $\begin{array}{l}\text { Phase II } \\
\text { Randomised, double-blind, } \\
\text { placebo-controlled } \\
\text { Topical Isopropyl unoprostone } \\
\text { Placebo }\end{array}$ & $\begin{array}{l}\mathrm{RP} \text { (clinical } \\
\text { diagnosis) }\end{array}$ & $\begin{array}{l}\text { MP-1 } \\
\text { Mesopic } \\
\text { No DA } \\
24 \text { points } \\
10^{\circ} \text { grid } \\
4-2 \text { double staircase }\end{array}$ & $\begin{array}{l}\text { Primary } \\
\text { Change in central } 2^{\circ} \text { retinal sensitivity } \\
\text { (specifically No. of points with } \geq 4 \mathrm{~dB} \\
\text { decrease) } \\
\text { Secondary } \\
\text { BCVA } \\
\text { CS } \\
\text { Change in } 10^{\circ} \text { retinal sensitivity } \\
\text { Mean deviation in HFA } \\
\text { QoL }\end{array}$ \\
\hline Tawada et al. [208], 2013 & $\begin{array}{l}\text { Non-comparative pilot study } \\
\text { Topical isopropyl unoprostone }\end{array}$ & $\begin{array}{l}\mathrm{RP}(\text { clinical } \\
\text { diagnosis) }\end{array}$ & $\begin{array}{l}\text { MP-1 } \\
\text { Mesopic } \\
\text { No DA } \\
10^{\circ}(24 \text { points }) \\
4-2 \text { staircase } \\
3^{\circ} \text { red single cross fixation target }\end{array}$ & $\begin{array}{l}\text { Primary } \\
\text { Change to central } 2^{\circ} \text { retinal sensitivity } \\
\text { Secondary } \\
\text { BCVA } \\
\text { MP (MS; central } 10^{\circ} \mathrm{MS} \text {; No. of points } \\
\text { improved by } \geq 2 \text { and } \geq 4 \mathrm{~dB} \text { ) } \\
\text { Perimetry (MD on HFA } 10-2 \text { ) }\end{array}$ \\
\hline $\begin{array}{l}\text { Wagner et al. [209], } 2017 \\
\text { NCT01847365 }\end{array}$ & $\begin{array}{l}\text { Single-arm open label interven- } \\
\text { tional safety trial } \\
\text { Weekly transcorneal electrical } \\
\text { stimulation }\end{array}$ & $\begin{array}{l}\mathrm{RP} \text { (varying } \\
\text { genotypes) }\end{array}$ & $\begin{array}{l}\text { MAIA } \\
\text { Mesopic } \\
20-\text { min DA } \\
10-2 \text { grid }\end{array}$ & $\begin{array}{l}\text { Primary } \\
\text { Safety } \\
\text { Secondary } \\
\text { Efficacy according to structure and } \\
\text { function (BCVA, MP [MS] or Goldmann } \\
\text { VF) }\end{array}$ \\
\hline $\begin{array}{l}\text { Campochiaro et al. [210], } 2020 \\
\text { NCT03063021 }\end{array}$ & $\begin{array}{l}\text { Phase I } \\
\text { Oral } N \text {-acetylcysteine }\end{array}$ & $\begin{array}{l}\mathrm{RP} \text { (clinical } \\
\text { diagnosis, } \\
\text { varying } \\
\text { genotypes) }\end{array}$ & $\begin{array}{l}\text { MAIA } \\
\text { Mesopic } \\
68 \text { points } \\
10-2 \text { grid }\end{array}$ & $\begin{array}{l}\text { Primary } \\
\text { Safety, tolerance } \\
\text { Secondary } \\
\text { BCVA, MS, EZ width, aqueous NAC }\end{array}$ \\
\hline $\begin{array}{l}\text { Kong et al. [211], } 2020 \\
\text { NCT03063021 (this is a point } \\
\text { wise analysis of data from } \\
\text { Campochiaro et al. [210]) }\end{array}$ & $\begin{array}{l}\text { Phase I } \\
\text { Dose escalation, single-centre, } \\
\text { open-label } \\
\text { Oral } N \text {-acetylcysteine }\end{array}$ & $\begin{array}{l}\mathrm{RP} \text { (clinical } \\
\text { diagnosis, } \\
\text { varying } \\
\text { genotypes) }\end{array}$ & $\begin{array}{l}\text { MAIA } \\
\text { Mesopic } \\
68 \text { points } \\
\text { Central } 20^{\circ}\end{array}$ & $\begin{array}{l}\text { Primary } \\
\text { Safety } \\
\text { Secondary } \\
\text { Visual function: BCVA, } \\
\text { MP - PWS change } \geq 6 \mathrm{~dB} \text {; } \\
\text { PWS analysed according to location: } \\
\text { foveal }\left(2^{\circ}\right) \text {, perifoveal }\left(4^{\circ}\right) \text {, peripheral } \\
\left(\text { remaining } 6-10^{\circ}\right) \text {; superior/inferior, } \\
\text { temporal/nasal areas }\end{array}$ \\
\hline
\end{tabular}


Table 5 (continued)

\begin{tabular}{|c|c|c|c|c|}
\hline Study & Study design and interventions & Condition & Microperimetry test parameters & Endpoints \\
\hline Chen et al. [212], 2008 & $\begin{array}{l}\text { Pilot study } \\
\text { Autologous RPE-choroid graft } \\
\text { subfoveally }\end{array}$ & Macular dystrophy & $\begin{array}{l}\text { MP-1 } \\
\text { Mesopic } \\
\text { Custom grid (postop. grid to include graft } \\
\text { and surrounding area) } \\
4-2 \text { staircase }\end{array}$ & $\begin{array}{l}\text { Primary } \\
\text { Safety, presence of retinal function } \\
\text { Secondary } \\
\text { PWS } \\
\text { Maximal point sensitivity FAF } \\
\text { BCVA } \\
\text { Reading acuity } \\
\text { CS }\end{array}$ \\
\hline $\begin{array}{l}\text { Park et al. [213], } 2015 \\
\text { NCT01736059 }\end{array}$ & $\begin{array}{l}\text { Phase I } \\
\text { Intravitreal autologous CD } 34+ \\
\text { bone marrow stem cells }\end{array}$ & $\begin{array}{l}\text { Ischaemic and } \\
\text { degenerative } \\
\text { retinal conditions } \\
\text { (RVO, AMD, } \\
\text { STGD, RP) }\end{array}$ & $\begin{array}{l}\text { MAIA } \\
\text { Mesopic } \\
\text { Standard } 10^{\circ} \text { (37 points) }\end{array}$ & $\begin{array}{l}\text { Primary } \\
\text { AEs, no of CD } 34+\text { cells isolated and } \\
\text { injected } \\
\text { Secondary } \\
\text { MP - MS and \% reduced sensitivity } \\
\text { Changes in Goldmann perimetry, FFA, } \\
\text { ERG, OCT, AO-OCT }\end{array}$ \\
\hline
\end{tabular}

Unless otherwise stated, test parameters involved stimuli of Goldmann III size and 200 ms duration. AAV, recombinant adeno-associated virus (vector used for gene therapy) AAV2.REP1; AE, adverse events; AO-OCT, adaptive optics optical coherence topography; CNV, choroidal neovascularisation; CS, contrast sensitivity; DA, dark adaption; ERG, electroretinography; FFA, fundus fluorescein angiogram; FST, full-field stimulus testing; hESC-RPE, human embryonic stem cell-derived retinal pigment epithelium; HFA, Humphrey field analyser; MD, mean deviation; mERG, multifocal ERG; NAC, N-acetylcysteine; pts, patients; PWS, pointwise sensitivity; QoL, quality of life measures/questionnaires; RP, retinitis pigmentosa; RPE, retinal pigment epithelium; RPGR RP, X-linked retinitis pigmentosa secondary to RPGR defect; RVO, retinal vein occlusion; STGD1, Stargardt disease type 1; VF, visual field; VFMA, visual field modelling and analysis; XLRS, X-linked retinoschisis.

\section{Inherited Retinal Disease}

This resulted in the identification of 22 publications relating to interventional studies for IRD. Of these, 1 was excluded on account of it being a description of a single patient. From the remainder, there were 6 publications on choroideremia, 3 on Leber congenital amaurosis (LCA), and 1 interventional study each for X-linked retinoschisis, $\mathrm{X}$-linked retinitis pigmentosa due to a defect in $R P G R$, CNGA3-related achromatopsia, autosomal dominant drusen, and Stargardt disease. Five studies involved interventions in patients with retinitis pigmentosa of various genotypes, whilst one study involved patients with macular dystrophy and another described intervention in a mixed patient group including IRD. The studies are summarised in Table 5 and a selection of these will be discussed in further detail.

\section{Choroideremia}

Choroideremia is the ongoing target of ocular gene therapy intervention and numerous phase I/II trials have published their results [28-30, 107]. As standard for phase I/II trials, changes in BCVA and the occurrence of serious adverse events (SAE) are predominantly cited as primary endpoints. Mesopic MP using the MAIA features prominently as a secondary endpoint. Interestingly, its use in some trials has taken the characteristics of the underlying condition into account by using customised grids [28, 107].

Microperimetry: Trial Endpoint in Retinal Disease
A typical feature of choroideremia is the presence of scalloped patches of choroidal atrophy in the peripheral retina. These atrophic patches gradually enlarge, coalesce, and encroach in a distinctive centripetal fashion on a central island of functioning retina. These areas can be tracked using FAF imaging where they appear hypofluorescent due to complete loss of overlying RPE [108]. In one phase I/II gene therapy study, intact areas of retina were identified pre-intervention by FAF and MP grids created to fit these areas [107]. Alternatively, or in combination with custom grids, standard grids of varying degrees of coverage have been used within the same trial, the choice dependent on the size of the residual functioning retina within the same trial $[28,29,109]$. In studies comprising small numbers of participants with differing disease severity (and thus varying areas of intact retina), this tailored approach has its advantages. However, this does have implications for direct comparisons between eyes, between patients, and across studies, and as the number of tested points vary, in averaging to obtain MS.

No statistically significant changes in retinal sensitivity measures in treated eyes versus untreated eyes or to baseline were demonstrated in these studies, although trends towards improvement in treated eyes were suggested from increases in BCVA and retinal sensitivity in some [107]. A range of additional parameters that were also explored included "peak" retinal sensitivity and the total number of test points seen [107], thus demonstrating the interest in defining additional metrics derived from analysis of the raw data to better determine intervention effects. 
Table 6. Summary of randomised interventional studies in acquired retinal disease and their endpoints

\begin{tabular}{|c|c|c|c|c|}
\hline Study & Study design and interventions & Condition & Microperimetry test parameters & Endpoints \\
\hline $\begin{array}{l}\text { Weigert et al. } 2011 \\
\text { [146] NCT00879671 }\end{array}$ & $\begin{array}{l}\text { Single-centre RCT } \\
\text { Lutein } \\
\text { Placebo }\end{array}$ & $\begin{array}{l}\text { Age-related macular } \\
\text { degeneration. (AREDS } \\
\text { stages 2, 3, and } 4 \text { with no } \\
\text { CNV) }\end{array}$ & $\begin{array}{l}\text { MP-1 } \\
\text { Mesopic } \\
41 \text { stimuli } \\
12^{\circ} \text { grid } \\
4-2-1 \text { staircase } \\
3^{\circ} \text { red cross fixation target }\end{array}$ & $\begin{array}{l}\text { Primary } \\
\text { MPOD } \\
\text { Secondary } \\
\text { BCVA } \\
\text { MS }\end{array}$ \\
\hline $\begin{array}{l}\text { Huang et al. [79], } 2015 \\
\text { NCT10528605 }\end{array}$ & $\begin{array}{l}\text { Single-centre RCT } \\
10 \mathrm{mg} \text { lutein } \\
20 \mathrm{mg} \text { lutein } \\
10 \mathrm{mg} \text { lutein + zeaxanthin } \\
\text { Placebo }\end{array}$ & $\begin{array}{l}\text { AREDS classified early } \\
\text { age-related macular } \\
\text { degeneration }\end{array}$ & $\begin{array}{l}\text { MP-1 } \\
\text { Mesopic } \\
10-\text { min DA } \\
41 \text { stimuli } \\
10^{\circ} \text { grid } \\
4-2-1 \text { staircase } \\
3^{\circ} \text { red cross fixation target }\end{array}$ & $\begin{array}{l}\text { Primary } \\
\text { MPOD } \\
\text { Secondary } \\
\text { MfERG (assessed at } 0 \text { and } 48 \text { weeks only) } \\
\text { MS (assessed at } 48 \text { weeks and } 24 \text { months only) } \\
1^{\circ} \mathrm{MS} \\
3^{\circ} \mathrm{MS} \\
5^{\circ} \mathrm{MS}\end{array}$ \\
\hline Dow et al. [96], 2016 & $\begin{array}{l}\text { Single-centre RCT } \\
\text { Oral telomerase (TA-65) } \\
\text { Placebo }\end{array}$ & $\begin{array}{l}\text { Early age-related } \\
\text { macular degeneration }\end{array}$ & $\begin{array}{l}\text { MAIA } \\
\text { Mesopic } \\
61 \text { stimuli } \\
10^{\circ} \text { grid } \\
4-2 \text { staircase } \\
1^{\circ} \text { red circle fixation target }\end{array}$ & $\begin{array}{l}\text { Primary } \\
\text { \% reduced threshold points } \\
\text { MS }\end{array}$ \\
\hline $\begin{array}{l}\text { Wu et al. [150], } 2019 \\
\text { NCT01790802 }\end{array}$ & $\begin{array}{l}\text { Multicentre RCT } \\
\text { SNL } \\
\text { Sham laser }\end{array}$ & $\begin{array}{l}\text { Intermediate age-related } \\
\text { macular degeneration }\end{array}$ & $\begin{array}{l}\text { MAIA } \\
\text { Mesopic } \\
37 \text { stimuli } \\
12^{\circ} \text { grid }\end{array}$ & $\begin{array}{l}\text { Secondary } \\
\text { Time to develop late AMD in non-study eye } \\
\text { Exploratory } \\
\text { Rate of change in: } \\
\text { BCVA } \\
\text { LLVA } \\
\text { MS } \\
\text { Drusen volume } \\
\text { Night vision questionnaire } \\
\text { Impact of visual impairment questionnaire }\end{array}$ \\
\hline $\begin{array}{l}\text { Sacu et al. [147], } 2008 \\
\text { EudraCT No. } \\
\text { 2005-000776-41 }\end{array}$ & $\begin{array}{l}\text { Single-centre RCT } \\
\text { Standard PDT } \\
\text { Reduced fluence PDT }\end{array}$ & $\begin{array}{l}\text { Neovascular age-related } \\
\text { macular degeneration }\end{array}$ & $\begin{array}{l}\text { MP-1 } \\
\text { Mesopic } \\
41 \text { stimuli } \\
12^{\circ} \text { grid } \\
4-2-1 \text { staircase } \\
5^{\circ} \text { cross fixation target }\end{array}$ & $\begin{array}{l}\text { Primary } \\
\text { BCVA } \\
\text { Secondary } \\
\text { CRT } \\
\text { MS }\end{array}$ \\
\hline $\begin{array}{l}\text { Dunavoelgyi } \\
\text { et al. [97], } 2011 \\
\text { EudraCT No. } \\
\text { 2005-000776-41 }\end{array}$ & $\begin{array}{l}\text { Single-centre RCT } \\
\text { Standard PDT } \\
\text { Reduced fluence PDT }\end{array}$ & $\begin{array}{l}\text { Neovascular age-related } \\
\text { macular degeneration }\end{array}$ & $\begin{array}{l}\text { MP-1 } \\
\text { Mesopic } \\
41 \text { stimuli } \\
12^{\circ} \text { grid } \\
4-2-1 \text { staircase } \\
5^{\circ} \text { cross fixation target }\end{array}$ & $\begin{array}{l}\text { Primary } \\
\text { MS } \\
\text { Relative scotoma size ( } \% \text { of points }<10 \mathrm{~dB} \text { ) } \\
\text { Absolute scotoma size ( } \% \text { of points }<0 \mathrm{~dB} \text { ) } \\
\text { Secondary } \\
\text { BCVA } \\
\text { FA }\end{array}$ \\
\hline $\begin{array}{l}\text { Rezar-Dreindl } \\
\text { et al. [148], } 2017 \\
\text { NCT01162746 }\end{array}$ & $\begin{array}{l}\text { Single-centre RCT } \\
\text { RM } \\
\text { RM + DEX }\end{array}$ & $\begin{array}{l}\text { Neovascular age-related } \\
\text { macular degeneration }\end{array}$ & $\begin{array}{l}\text { MP-1 } \\
\text { Mesopic } \\
4-2-1 \text { staircase } \\
3^{\circ} \text { circle fixation target }\end{array}$ & $\begin{array}{l}\text { Primary } \\
\text { Time until RM retreatments } \\
\text { Total number of RM retreatments } \\
\text { Secondary } \\
\text { BCVA } \\
\text { CFT } \\
\text { MS }\end{array}$ \\
\hline Limoli et al. [152], 2018 & $\begin{array}{l}\text { Single-centre pilot RCT } \\
\text { Suprachoroidal autologous graft } \\
\text { Control }\end{array}$ & $\begin{array}{l}\text { Dry age-related macular } \\
\text { degeneration }\end{array}$ & MAIA & $\begin{array}{l}\text { BCVA } \\
\text { MS }\end{array}$ \\
\hline $\begin{array}{l}\text { Heier et al. [98], } 2020 \\
\text { NCT02247479 } \\
\text { NCT02247531 }\end{array}$ & $\begin{array}{l}\text { Subgroup of phase } 3 \text { multicentre } \\
\text { RCT } \\
\text { Lampalizumab q4w } \\
\text { Lampalizumab q6w } \\
\text { Sham }\end{array}$ & $\begin{array}{l}\text { Bilateral geographic } \\
\text { atrophy }\end{array}$ & $\begin{array}{l}\text { MP1 } \\
\text { Mesopic } \\
68 \text { stimuli } \\
10-2 \text { grid } \\
4-2 \text { staircase }\end{array}$ & $\begin{array}{l}\text { Primary } \\
\text { GA lesion size on FAF } \\
\text { Secondary } \\
\text { BCVA } \\
\text { LLVA } \\
\text { Reading speed } \\
\text { MS } \\
\text { Number of absolute scotoma points }(<0 \mathrm{~dB}) \\
\text { FRI } \\
\text { NEIVFQ-25 }\end{array}$ \\
\hline
\end{tabular}


Table 6 (continued)

\begin{tabular}{|c|c|c|c|c|}
\hline Study & Study design and interventions & Condition & Microperimetry test parameters & Endpoints \\
\hline $\begin{array}{l}\text { Rinaldi et al. [151], } 2017 \\
\text { NCT01968486 }\end{array}$ & $\begin{array}{l}\text { Single-centre RCT } \\
\text { Verteporfin + standard } \\
\text { fluence PDT } \\
\text { Verteporfin + Reduced } \\
\text { Fluence + RM } \\
\text { RM }\end{array}$ & $\begin{array}{l}\text { Myopic choroidal } \\
\text { neovascularisation }\end{array}$ & $\begin{array}{l}\text { MP-1 } \\
\text { Mesopic } \\
45 \text { stimuli } \\
12^{\circ} \text { grid } \\
4-2 \text { strategy }\end{array}$ & $\begin{array}{l}\text { Primary } \\
\text { BCVA } \\
\text { CRT } \\
\text { Secondary } \\
\text { Number of RM retreatments } \\
\text { Time to first RM retreatment } \\
\text { FA and OCT anatomical changes } \\
\text { Exploratory } \\
\text { MS }\end{array}$ \\
\hline $\begin{array}{l}\text { Chew et al. [149], } 2019 \\
\text { Effect of ciliary } \\
\text { neurotrophic factor on } \\
\text { retinal neurodegeneration } \\
\text { in patients with macular } \\
\text { telangiectasia type } 2 \\
\text { NCT01949324 }\end{array}$ & $\begin{array}{l}\text { Multicentre RCT } \\
\text { CNTF implant surgery } \\
\text { Sham surgery }\end{array}$ & $\begin{array}{l}\text { Macular telangiectasia } \\
\text { type } 2\end{array}$ & MAIA & $\begin{array}{l}\text { Primary } \\
\text { EZ disruption on SD OCT at } 24 \text { months } \\
\text { Secondary } \\
\text { EZ disruption on SD OCT at } 12 \text { months } \\
\text { ETDRS letters } \\
\text { MS } \\
\text { Monocular reading speed } \\
30-2 \text { Humphrey visual fields } \\
\text { Post hoc } \\
\text { Aggregate sensitivity loss }\end{array}$ \\
\hline $\begin{array}{l}\text { Vujosevic et al. [80], } \\
2010\end{array}$ & $\begin{array}{l}\text { Single-centre RCT } \\
\text { SMDL } \\
\text { ETDRS laser }\end{array}$ & $\begin{array}{l}\text { Diabetic macular } \\
\text { oedema }\end{array}$ & $\begin{array}{l}\text { MP-1 } \\
\text { Mesopic } \\
5 \text {-min DA } \\
45 \text { stimuli } \\
12^{\circ} \text { radial grid } \\
4-2-1 \text { staircase } \\
1^{\circ} \text { red ring fixation target }\end{array}$ & $\begin{array}{l}\text { Primary } \\
4^{\circ} \text { FMS } \\
\text { MS } \\
\text { FAF } \\
\text { Secondary } \\
\text { ETDRS letters } \\
\text { CRT }\end{array}$ \\
\hline $\begin{array}{l}\text { Vujosevic et al. [81], } \\
2015\end{array}$ & $\begin{array}{l}\text { Single-centre pilot RCT } \\
\text { Yellow micropulse laser } \\
\text { Infrared micropulse laser }\end{array}$ & $\begin{array}{l}\text { Diabetic macular } \\
\text { oedema }\end{array}$ & $\begin{array}{l}\text { MP-1 } \\
\text { Mesopic } \\
5 \text {-min DA } \\
45 \text { stimuli } \\
\text { Custom } 12^{\circ} \text { grid } \\
4-2 \text { staircase } \\
1^{\circ} \text { red ring fixation target }\end{array}$ & $\begin{array}{l}\text { Primary: } \\
\text { BCVA } \\
4^{\circ} \mathrm{FMS} \\
\text { MS } \\
\text { Structural parameters on OCT }\end{array}$ \\
\hline $\begin{array}{l}\text { Gonzalez et al. [164], } 2015 \\
\text { NCT00789477 }\end{array}$ & $\begin{array}{l}\text { Sub-group of multicentre ran- } \\
\text { domised, double-masked phase } 2 \\
\text { studies }[166,214] \\
\text { Laser } \\
0.5 \text { q4 IA } \\
\text { 2q4 IAI } \\
2 \text { q8 IA } \\
2 \text { PRN }\end{array}$ & $\begin{array}{l}\text { Diabetic macular } \\
\text { oedema }\end{array}$ & $\begin{array}{l}\text { MP-1 } \\
\text { Mesopic } \\
5 \text {-min DA } \\
29 \text { stimuli } \\
16^{\circ} \text { grid } \\
4-2-1 \text { strategy } \\
1^{\circ} \text { red ring fixation target }\end{array}$ & $\begin{array}{l}\text { Primary } \\
\text { BCVA at } 24 \text { weeks } \\
\text { Secondary } \\
\text { BCVA at } 52 \text { weeks } \\
\% \text { subjects with } 15 \text { letter gain } \\
\text { CRT } \\
\text { number of laser treatments } \\
\text { MS in OCT subfields } \\
\text { Central } 4^{\circ}\left(2^{\circ} \text { radius }\right) \\
\text { Inner } 10^{\circ}\left(5^{\circ} \text { radius }\right) \\
\text { Inner to outer ring }\left(2-8^{\circ} \text { radius }\right) \\
\text { FS }\end{array}$ \\
\hline $\begin{array}{l}\text { Comyn et al. [82], } 2014 \\
\text { NCT01223612 }\end{array}$ & $\begin{array}{l}\text { Single-centre RCT } \\
2-1 \text { randomisation } \\
\text { RM } \\
\text { ETDRS laser }\end{array}$ & $\begin{array}{l}\text { Diabetic macular } \\
\text { oedema }\end{array}$ & $\begin{array}{l}\text { MP-1 } \\
\text { Mesopic } \\
45 \text { stimuli } \\
\text { Custom } 12^{\circ} \text { grid }\end{array}$ & $\begin{array}{l}\text { Primary } \\
\text { Adverse events } \\
\text { BCVA } \\
\text { FA } \\
\text { Exploratory functional } \\
4^{\circ} \text { FMS } \\
\text { MS } \\
\text { Colour contrast thresholds } \\
\text { Electrophysiology parameters }\end{array}$ \\
\hline $\begin{array}{l}\text { Mylonas et al. [165], } \\
2020 \\
\text { NCT00682539 }\end{array}$ & $\begin{array}{l}\text { Single-centre prospective } \\
\text { randomised study } \\
\text { Bevacizumab } \\
\text { Triamcinolone }\end{array}$ & $\begin{array}{l}\text { Diabetic macular } \\
\text { oedema }\end{array}$ & $\begin{array}{l}\text { MP-1 } \\
\text { Mesopic } \\
41 \text { stimuli } \\
12^{\circ} \text { grid } \\
4-2-1 \text { strategy } \\
3^{\circ} \text { red cross fixation target }\end{array}$ & $\begin{array}{l}\text { Primary } \\
\text { BCVA } \\
\text { CRT } \\
\text { (presented in Kriechbaum, } 2014[167]) \\
\text { Secondary } \\
\text { MS } \\
\text { No. of absolute scotoma points }(<0 \mathrm{~dB}) \\
\text { Absolute scotoma size (\% of absolute scotoma } \\
\text { points) } \\
\text { Relative scotoma size (\% of relative scotoma } \\
\text { points } 1 \text { and }<10 \mathrm{~dB})\end{array}$ \\
\hline Forte e al. [215], 2011 & $\begin{array}{l}\text { Single-centre RCT } \\
\text { Flavonoid supplement } \\
\text { Control }\end{array}$ & $\begin{array}{l}\text { Diabetic cystoid } \\
\text { macular oedema } \\
\text { without macular } \\
\text { thickening }\end{array}$ & $\begin{array}{l}\text { SD-SLO/OCT } \\
\text { Mesopic } \\
8^{\circ} \text { grid } \\
4-2-1 \text { staircase }\end{array}$ & $\begin{array}{l}\text { BCVA } \\
\text { CRT } \\
\text { MS } \\
\text { FS }\end{array}$ \\
\hline
\end{tabular}


Table 6 (continued)

\begin{tabular}{|c|c|c|c|c|}
\hline Study & Study design and interventions & Condition & Microperimetry test parameters & Endpoints \\
\hline $\begin{array}{l}\text { Wallsh et al. [179], } 2016 \\
\text { NCT01449682 }\end{array}$ & $\begin{array}{l}\text { Exploratory single-centre RCT } \\
\text { DEX 4-month regime } \\
\text { DEX PRN regime }\end{array}$ & $\begin{array}{l}\text { Macular oedema } \\
\text { secondary to retinal } \\
\text { vein occlusion }\end{array}$ & MP-1 & $\begin{array}{l}\text { Primary } \\
\text { Multifocal ERG } \\
\text { MS } \\
\text { Secondary } \\
\text { OCT } \\
\text { BCVA }\end{array}$ \\
\hline $\begin{array}{l}\text { Mackensen et al. [216], } \\
2013 \\
\text { NCT00344253 }\end{array}$ & $\begin{array}{l}\text { Single-centre RCT } \\
\text { Interferon } \\
\text { Methotrexate }\end{array}$ & $\begin{array}{l}\text { Macular oedema in } \\
\text { uveitis (primary or } \\
\text { associated with multiple } \\
\text { sclerosis) }\end{array}$ & $\begin{array}{l}\text { MP-1 } \\
\text { Mesopic } \\
10^{\circ} \text { grid }\end{array}$ & $\begin{array}{l}\text { Primary } \\
\text { Change in BCVA } \\
\text { Secondary } \\
\text { CRT } \\
\text { Inflammatory activity } \\
\text { MS } \\
\text { NEIVFQ-25 } \\
\text { SF36 }\end{array}$ \\
\hline $\begin{array}{l}\text { van Dijk et al. [173], } \\
2018 \\
\text { NCT01797861 }\end{array}$ & $\begin{array}{l}\text { Multicentre RCT } \\
\text { Half-dose PDT } \\
\text { HSML }\end{array}$ & $\begin{array}{l}\text { Chronic central serous } \\
\text { chorioretinopathy }\end{array}$ & $\begin{array}{l}\text { MP-1 and MAIA } \\
\text { "to a standard protocol" [217] } \\
\text { (threshold measurements from each } \\
\text { device converted to a single scale as } \\
\text { per Parodi et al. [63]) }\end{array}$ & $\begin{array}{l}\text { Primary } \\
\text { Absence of SRF at } 6-8 \text { weeks } \\
\text { Secondary } \\
\text { Absence of SRF at } 7-8 \text { months } \\
\text { No. of repeat treatments required } \\
\text { ETDRS letters } \\
\text { MS } \\
\text { NEIVFQ-25 }\end{array}$ \\
\hline $\begin{array}{l}\text { van Rijssen et al. [174], } \\
2020 \\
\text { NCT01797861 }\end{array}$ & $\begin{array}{l}\text { Prospective crossover treatment } \\
\text { Multicentre RCT [173] } \\
\text { Crossover to Half dose PDT } \\
\text { Crossover to HSML }\end{array}$ & $\begin{array}{l}\text { Chronic central serous } \\
\text { chorioretinopathy }\end{array}$ & $\begin{array}{l}\text { MP-1 and MAIA } \\
\text { No other parameters provided [217] } \\
\text { (threshold measurements from each } \\
\text { device converted to single scale as per } \\
\text { Parodi et al. [63]) }\end{array}$ & $\begin{array}{l}\text { Primary } \\
\text { Resolution of SRF } \\
\text { Secondary } \\
\text { ETDRS letters } \\
\text { MS } \\
\text { NEIVFQ-25 }\end{array}$ \\
\hline Dang.et al. [175], 2013 & $\begin{array}{l}\text { Single-centre RCT } \\
\text { H. Pylori treatment } \\
\text { Placebo }\end{array}$ & $\begin{array}{l}\text { Central serous } \\
\text { chorioretinopathy } \\
\text { (positive for H. pylori) }\end{array}$ & $\begin{array}{l}\text { MP-1 } \\
\text { Mesopic } \\
33 \text { stimuli } \\
15^{\circ} \text { grid } \\
4-2-1 \text { staircase }\end{array}$ & $\begin{array}{l}\text { Primary } \\
\text { Resolution rate of SRF } \\
\text { Secondary } \\
\text { BCVA } \\
\text { MS }\end{array}$ \\
\hline $\begin{array}{l}\text { Viana et al. [177], } 2020 \\
\text { Brazilian clinical trial No. } \\
\text { RBR-3wmd9s }\end{array}$ & $\begin{array}{l}\text { Single-centre RCT } \\
\text { PPV + phacoemulsification } \\
\text { PPV deferred phacoemulsification }\end{array}$ & $\begin{array}{l}\text { Full-Thickness Macular } \\
\text { hole }\end{array}$ & $\begin{array}{l}\text { MAIA } \\
\text { Mesopic } \\
37 \text { stimuli } \\
6^{\circ} \text { grid } \\
4-2 \text { staircase }\end{array}$ & $\begin{array}{l}\text { Primary } \\
\text { logMAR BCVA change from baseline } \\
\text { Secondary } \\
\text { MS } \\
\% \text { closure rate }\end{array}$ \\
\hline $\begin{array}{l}\text { Morescalchi et al. [83], } \\
2020 \\
\text { NCT02361645 }\end{array}$ & $\begin{array}{l}\text { Single-centre prospective, } \\
\text { randomised, comparative } \\
\text { study } \\
\text { Complete ILM peel } \\
\text { Foveal-sparing ILM peel } \\
\text { 12-month follow-up }\end{array}$ & Macular hole $>250 \mu$ & $\begin{array}{l}\text { OPKO/OTI } \\
\text { Mesopic } \\
28 \text { stimuli } \\
\text { Polar } 3-12^{\circ} \text { grid }\end{array}$ & $\begin{array}{l}\text { Primary } \\
\text { ETDRS letters } \\
\text { Secondary } \\
\text { CRT } \\
\text { FMS (mean of } 4 \text { central points) } \\
\text { Adverse events }\end{array}$ \\
\hline $\begin{array}{l}\text { Morescalchi et al. [84], } \\
2020 \\
\text { NCT02361645 }\end{array}$ & $\begin{array}{l}\text { Single-centre RCT } \\
\text { Foveal-sparing ILM peel } \\
\text { Control }\end{array}$ & $\begin{array}{l}\text { Degenerative lamellar } \\
\text { hole }\end{array}$ & $\begin{array}{l}\text { OPKO/OTI } \\
\text { Mesopic } \\
28 \text { stimuli } \\
\text { Polar } 3-12^{\circ} \text { grid }\end{array}$ & $\begin{array}{l}\text { Primary } \\
\text { ETDRS letters } \\
\text { Secondary } \\
\text { CRT } \\
\text { FMS (mean of } 4 \text { central points) } \\
\text { Structural endpoints } \\
\text { Adverse events }\end{array}$ \\
\hline $\begin{array}{l}\text { Russo et al. [85], } 2019 \\
\text { NCT02361645 }\end{array}$ & $\begin{array}{l}\text { Single-centre prospective, ran- } \\
\text { domised, comparative study } \\
\text { Complete ILM peel } \\
\text { Foveal-sparing ILM peel }\end{array}$ & Epiretinal membrane & $\begin{array}{l}\text { OPKO/OTI } \\
\text { Mesopic } \\
28 \text { stimuli } \\
\text { Polar } 3^{\circ} \text { to } 12^{\circ} \text { grid }\end{array}$ & $\begin{array}{l}\text { Primary } \\
\text { ETDRS letters } \\
\text { Secondary } \\
\text { CRT } \\
\text { FMS (mean of } 4 \text { central points) } \\
\text { PMS (mean of peripheral } 24 \text { points) } \\
\text { Adverse events }\end{array}$ \\
\hline Eissa et al. [86], 2018 & $\begin{array}{l}\text { Single-centre prospective } \\
\text { interventional randomised } \\
\text { comparative study } \\
\text { With ILM peeling } \\
\text { Without ILM peeling }\end{array}$ & $\begin{array}{l}\text { Macula-off rhegmatog- } \\
\text { enous retinal detachment }\end{array}$ & $\begin{array}{l}\text { OPKO/OTI } \\
\text { Mesopic } \\
56 \text { stimuli } \\
10-2 \text { grid } \\
4-2 \text { staircase }\end{array}$ & $\begin{array}{l}\text { Primary } \\
\text { BCVA } \\
\text { MS } \\
2^{\circ} \text { MS (mean of } 4 \text { central points) } \\
\text { Secondary } \\
\text { OCT features }\end{array}$ \\
\hline
\end{tabular}


Table 6 (continued)

\begin{tabular}{|c|c|c|c|c|}
\hline Study & Study design and interventions & Condition & Microperimetry test parameters & Endpoints \\
\hline Ripandelli et al. [87], 2015 & $\begin{array}{l}\text { Multicentre RCT } \\
\text { ILM peel } \\
\text { No ILM peel }\end{array}$ & $\begin{array}{l}\text { Idiopathic macular } \\
\text { pucker }\end{array}$ & $\begin{array}{l}\text { MP-1 } \\
\text { Mesopic } \\
5 \text {-min DA } \\
33 \text { stimuli } \\
12^{\circ} \text { grid } \\
4-2-1 \text { staircase } \\
4^{\circ} \text { red cross fixation target }\end{array}$ & $\begin{array}{l}\text { Primary } \\
4^{\circ} \mathrm{MS} \\
12^{\circ} \mathrm{MS} \\
\text { Number of absolute scotoma points } \\
\text { Secondary } \\
\text { BCVA } \\
\text { OCT parameters }\end{array}$ \\
\hline Romano et al. [218], 2018 & $\begin{array}{l}\text { Single-centre prospective, } \\
\text { randomised, comparative } \\
\text { study } \\
\text { Trypan blue } 0.15 \%+\text { brilliant } \\
\text { blue } 0.05 \%+\text { lutein } 2 \% \\
\text { Trypan blue } 0.15 \%+\text { brilliant } \\
\text { blue } 0.025 \%+\text { polyethylene } \\
\text { lycol } 33504 \% \\
\text { Indocyanine green } 0.05 \%\end{array}$ & $\begin{array}{l}\text { Idiopathic epiretinal } \\
\text { membrane }\end{array}$ & $\begin{array}{l}\text { MP-1 } \\
\text { Mesopic } \\
5 \text {-min DA } \\
61 \text { stimuli } \\
10^{\circ} \text { grid } \\
4-2 \text { strategy } \\
2^{\circ} \text { red cross fixation target }\end{array}$ & $\begin{array}{l}\text { BCVA } \\
\text { MS }\end{array}$ \\
\hline Pilotto et al. [219], 2011 & $\begin{array}{l}\text { Single-centre prospective } \\
\text { randomised study } \\
\text { Standard PDT } \\
\text { Bolus PDT }\end{array}$ & Choroidal haemangioma & $\begin{array}{l}\text { MP-1 } \\
\text { Mesopic } \\
10^{\circ} \text { grid centred on lesion } \\
2^{\circ} \text { ring fixation target }\end{array}$ & $\begin{array}{l}\text { Primary } \\
\text { BCVA defined as: } \\
\text { Stable }(1 \text { line }) \\
\text { Improved }(>1 \text { line }) \\
\text { Decreased }(<1 \text { line }) \\
\text { MS over treated area defined as: } \\
\text { Stable }(2 \mathrm{~dB}) \\
\text { Improved }(>2 \mathrm{~dB}) \\
\text { Decreased }(<2 \mathrm{~dB})\end{array}$ \\
\hline
\end{tabular}

Unless otherwise stated, test parameters involved stimuli of Goldmann III size and $200 \mathrm{~ms}$ duration. AREDS, age-related eye disease study; BCVA, best-corrected visual acuity; CFT, central foveal thickness; CMT, central macular thickness; CNFT, ciliary neurotrophic factor; CRT, central retinal thickness; DEX, dexamethasone; DHA, docosahexaenoic acid; ETDRS, early treatment of diabetic retinopathy study; EZ, ellipsoid zone; FA, fluorescein angiography; FAF, fundus autofluorescence; FMS, foveal mean sensitivity (dB); FRI, functional reading index; FS, fixation stability; FSP, foveal sparing; HSML, high-density subthreshold micropulse laser; IA, intravitreal aflibercept; ILM, inner limiting membrane; LP, laser photocoagulation; MDOP, macular pigment optical density; MfERG, multifocal electroretinogram; MS, mean sensitivity (dB); NEIVFQ-25, National Eye Institute Visual Function Questionnaire 25; PDT, photodynamic therapy; PMS, perifoveal retinal sensitivity; PPV, pars plana vitrectomy; RCT, randomised control trial; RM, ranibizumab; SD-OCT, spectral domain optical coherence topography; SMDL, subthreshold micropulse diode laser; SNL, subthreshold nanosecond laser; SRF, subretinal fluid.

\section{Leber Congenital Amaurosis}

Numerous independent groups have reported the outcomes of AAV-mediated subretinal gene therapy intervention for LCA caused by defects in the RPE65 gene [110-119]. One has reported MP findings as a secondary endpoint, utilising both central (68 points) and peripheral (55 points) MP-1 grids on each patient $[110,120]$. During surgery, the retinotomy was made along the superotemporal arcade with the resultant bleb achieving foveal involvement in 10 of the 12 patients. The peripheral grid was positioned between 4 and $20^{\circ}$ above fixation to cover the site of the retinotomy and its surrounding area. Changes in retinal sensitivity were reported according to the number of points which showed statistically significant improvement. Initial improvement in retinal sensitivity, assessed by MP, was demonstrated in 5 treated eyes but appeared to decline from 6 to 12 months. A similar trend in dark-adapted perimetry-derived sensitivity measures was seen in 6 treated eyes. Such findings led to a new vector being developed to enhance potency and the potential for longer-lasting effects [121].

Microperimetry: Trial Endpoint in Retinal Disease
The only other group (with the exception of a 1-patient account [122]) to report use of MP also used the MP-1 device, opting to report MS values and the number of microscotomas, defined as points which were $0 \mathrm{~dB}$ [118]. Both of these metrics were reported to be stable in both treated and untreated eyes. Other groups have emphasised other efficacy measures, such as perimetry (kinetic and static) and perimetry-defined hill-of-vision modelling metrics [119] or full-field sensitivity to assess retinal sensitivity over MP $[112,113]$.

Discussion on RPE65 LCA is not complete without reference to the first gene therapy product (Luxturna ${ }^{\circledR}$; voretigene neparvovec-rzyl) to gain FDA and European Medicines Agency approvals, and which is also available as National Health Service (NHS) treatment in the UK. In the initial stages, the primary endpoint was safety, and a wide range of tests were used to evaluate visual function as the secondary endpoint $[114,115]$. This included pupillary reflexes, nystagmus testing, perimetry, OCT changes, autofluorescence changes, full-field stimulus testing (FST), electroretinography, mobility test- 
ing, and functional magnetic resonance imaging. Notably, by phase III, the primary endpoint was the change in vision-guided mobility performance under differing light levels at 1 year [117]. Therefore in these studies, tests other than MP were critical, particularly visionguided mobility, given that nyctalopia is a pertinent feature of the disease.

\section{X-Linked Retinoschisis}

$\mathrm{X}$-linked retinoschisis is also the target of gene therapy, with intravitreal delivery favoured given the fragile condition of the retina and our literature search identified 1 study fulfilling our criteria. Cukras et al. [31] reported 18-month results of a phase I/II trial of intravitreal AAV8-RS1 in 9 patients. Safety and the occurrence of inflammation were the primary endpoints. MP was used as one of the ways to evaluate retinal function as a secondary endpoint, with the authors analysing the raw data from MP-1 mesopic tests to categorise individual grid points as "dense scotomatous" if the threshold sensitivity value was $<0 \mathrm{~dB}$, or "responding" if otherwise. Grid points were further categorised according to whether they were "extra-scotomatous" if separated from a dense scotomatous point by at least one other point, or "para-scotomatous" if immediately adjacent. Given the limited dynamic range of the MP-1 device and the occurrence of floor effects, this type of sub-categorisation allowed data to be meaningfully assessed. MS of responding points, extra-scotomatous and para-scotomatous points were separately reported but no significant changes were demonstrated.

\section{$X$-Linked Retinitis Pigmentosa due to RPGR Defect}

There are multiple ongoing clinical trials assessing the effect of ocular gene therapy for retinitis pigmentosa secondary to defects in the RPGR gene (subretinal delivery in NCT03252847, NCT03316560, NCT03116113; intravitreal delivery in NCT04517149). One trial (NCT03116113) has published preliminary results of up to 6 months of follow-up, for which MP has featured prominently and the current phase II/III of this trial has listed MP as a secondary endpoint [32]. Preliminary results of 18 patients included improvements in MS demonstrated in 6 patients under mesopic conditions using a standard 10-2 grid with the MAIA. Such gains were demonstrated in the medium and high vector dose cohorts, although the latter had a higher incidence ( 6 out of 9 patients in this cohort) of intraocular inflammation. MP results were presented using the standard device-generated interpolated colour "heat" maps of the sensitivity thresh- old at each tested point as well as a comparison of the number of points in which the stimulus was seen between treated/untreated eyes at baseline and at 6 months of follow-up. The course of inflammation in 1 high dose cohort patient was described in relation to the drop in MP retinal sensitivity value, corroborated by the patient's subjective symptoms of a paracentral scotoma and the development of subretinal hyperreflective lesions on OCT of presumed inflammatory origin. This was the clinical picture in the absence of a change in BCVA. Thus, MP helped to demonstrate efficacy as well as contribute to the clinical assessment of inflammatory complications in conjunction with other clinical findings.

Preliminary results from the $R P G R$ gene therapy trials have also been made available as press releases, notably one of which (NCT03316560) refers to obtaining additional clarification from the FDA regarding clinically meaningful improvements using MP [123]. Initially, the study group referred to responders as those who had shown an improvement beyond TRTV within the treated retinal area over at least 2 different visits (mesopic MP using MAIA) [124]. More recently, they have gone on to define responders as those demonstrating improvement of at least $7 \mathrm{~dB}$ in at least 5 points within the central area (centremost 36 points) of a 10-2 grid (consisting of a total of 68 points) [125].

\section{CNGA3 Achromatopsia}

Achromatopsia due to defects in CNGA3 is another area of active intense research in the field of ocular gene therapy, with multiple phase I/II trials taking place concurrentlyacrosstheworld(NCT03758404; NCT02935517; NCT02610582). All trials list safety and the incidence of treatment-related adverse events as their primary endpoints. Trial NCT03758404 lists broad secondary endpoints of changes to BCVA, perimetry, MP, and quality of life (QoL) measures. The secondary endpoints listed for trial NCT02935517 include changes to light aversion and colour vision. Although the secondary endpoints listed for NCT02610582 refer to changes in visual function, their recently published 12 -month follow-up results provide further details of the wide range of tests that have been used to characterise and monitor the patients' progress: BCVA; tests of spatial and temporal resolution; colour discrimination; flicker fusion frequency; FST to red stimuli; contrast sensitivity (CS); pupillary responses; QoL questionnaires, and MP [126]. The group report the absence of any substantial safety concerns and noted that 
all 9 adult patients received a subretinal injection of AAV8. CNGA3 demonstrated some improvement in at least 1 secondary endpoint test. There was a statistically significant mean increase in BCVA of 2.9 letters and a CS gain of $0.33 \log$ units in the treated eyes. However, MP changes (MS and fixation stability over 2 and $4^{\circ}$ ) were not significant. Aside from these MP findings, the trial investigators additionally utilised MP to track the PRL over time to confirm that at the 1-year follow-up, the PRL remains within the bleb boundaries of the treated macular area. Hill-of-vision analysis using the VFMA software has also been described in preliminary results with a modest improvement of $0.0613 \mathrm{~dB}$-sr in the central $10^{\circ}$ of the macula, but this was not statistically significant [93].

The use of such a broad range of tests to monitor the effect of the intervention is not an uncommon approach in such exploratory trials, given their early phase and the fact that many tests used to define clinical endpoints are yet to be fully established, including MP. In this trial, it is worth noting that the investigators also describe in detail how they set out to statistically combine 11 of the secondary endpoint tests to produce a single overall $Z$ score, individualised for each patient.

\section{Stargardt Disease}

Although not an interventional trial, the use of MP in the study of Stargardt disease has been significant and will be briefly mentioned here. Stargardt disease is the most common cause of inherited macular dystrophy, affecting around 1 in 8,000-100,000 people, with autosomal recessive mutations in $A B C A 4$ accounting for the most common subtype, Stargardt type I (STGD1) [27]. BCVA decline is slow, particularly in patients with older age at onset and thus other clinical endpoints to track early changes over time are being researched [127].

Structural metrics for disease progression include foveal outer retinal loss seen on OCT and changes to areas identified on FAF (typically a central area of hypofluorescence associated with RPE loss, surrounded by hyperfluorescence relating to lipofuscin accumulation) $[8,128]$. In terms of functional evaluation, MP is of particular interest given the typical eccentric fixation seen in this condition.

The Natural History of the Progression of Atrophy Secondary to Stargardt Disease (ProgStar) studies represent multicentre efforts to characterise and establish clinical endpoints for the condition. ProgStar consist of both retrospective and prospective longitudinal observational studies. To date they have reported findings from mesopic MP testing using the MP-1 in 359 eyes of 200 patients with STGD1 with 1 year of follow-up. A 10-2 grid of 68 points was used (with a customised spacing interval between points marginally distinct to that of the $2^{\circ}$ spacing of the Humphrey 10-2 grid).

ProgStar defines a deep scotomatous point as one in which retinal sensitivity was $0 \mathrm{~dB}$ or $<0 \mathrm{~dB}$. It is worth noting other studies such as the previously described trial by Cukras et al. [31] that defined absolute scotoma as $<0 \mathrm{~dB}$, as $0 \mathrm{~dB}$ still signifies a response, a distinction important for the interpretation of results. A relative scotoma was defined as a PWS value $>0 \mathrm{~dB}$ but $<12 \mathrm{~dB}$. Within these parameters, disease progression over 1 year was quantified in terms of decline in MS $(-0.68 \mathrm{~dB})$, increase in number of deep scotoma points ( +1.56 points), and a decrease in the number of points with a minimum retinal sensitivity of threshold of $12 \mathrm{~dB}$ ( -3.01 points). MS values were also provided for different grid subsections. Furthermore, custom software was used to automatically identify points adjacent to scotomas at the baseline visit to track over time [129]. This analysis revealed that the MS of points adjacent to scotomas undergo a faster rate of progression, highlighting their potential desirability as a clinical endpoint. It is thought that this may reflect higher disease activity occurring at the edges of the central atrophic area as it expands centrifugally. A previous ProgStar report had already established that a longer disease duration was associated with worse MS and a greater number of deep scotoma points [130].

Fixation metrics have also been studied in detail in the ProgStar studies and changes in location of PRL and fixation areas quantified over 1 year [104]. However, heterogeneity of changes were seen, perhaps reflecting the influence of neuronal adaptations that work to improve fixation, the existence of multiple PRLs, and the need for longer follow-up. Therefore, it was proposed that compared to retinal sensitivity, fixation metrics may be more suited as a secondary endpoint, with analysis focussed on a subset of patients.

The SMART (Scotopic Microperimetric Assessment of Rod Function in Stargardt Disease) is an ancillary study to ProgStar that focuses on evaluating scotopic function using the MP-1S. Data were collected from 118 eyes of 118 participants, although a different grid (composed of 40 points) was used for testing, thus limiting direct pointwise comparison with mesopic testing in these patients. MS in the first visit for mesopic and scotopic testing was 11.48 and $11.25 \mathrm{~dB}$, respectively. However, the annual 
rate of decline calculated from longitudinal data analysed over 2 years indicated that scotopic function deteriorated more than twice as quickly as mesopic function, with a loss of $1.42 \mathrm{~dB}$ compared to $0.63 \mathrm{~dB}$ per year, respectively $[131,132]$, and as such may be a more sensitive endpoint for future trials. Moreover, an earlier study of scotopic function in 12 STGD1 patients demonstrated an association between scotopic sensitivity loss and structural changes on SD-OCT and confocal OCT [133]. In some cases, areas with normal structure were also observed to have reduced scotopic sensitivity. In addition to studies of STGD1, the natural history of PROM-1-associated disease is currently being studied in ProgStar-4, adopting both mesopic and scotopic MP assessments [134].

\section{Acquired Retinal Disease}

Our review resulted in 32 randomised interventional studies in acquired retinal disease, précised in Table 6, broadly classified as 11 in acquired macular disease (9 AMD, 1 myopic CNV, and 1 macular telangiectasia), 7 in diabetic eye disease (6 diabetic macular oedema [DMO] and 1 non-proliferative diabetic retinopathy [NPDR]), 7 in vitreoretinal disease, 3 in CSCR, 3 in non-diabetic related macular oedema ( 2 branch retinal vein occlusion and 1 uveitic), and 1 choroidal haemangioma.

\section{Macular Degeneration}

MP has been used extensively in the study of macular disease in recent decades [135-137]. Although researchers in macular disease were amongst the first to recognise the benefits of MP, initially due to its compensation for poor fixation in GA [138], more recently its potential as an endpoint has moved centre stage $[6,12,13]$. Interest in mesopic and scotopic MP modalities has increased as their ability to capture subtle functional deficits in early and intermediate AMD [51, 59, 139-142] and quantify progression over time $[143,144]$ has been demonstrated. Of note, significant reductions in mesopic sensitivity over a period as short as 12 months have been shown [73, 145], implying MP endpoints may allow for shorter trial durations.

This review identified 11 randomised interventional studies in acquired macular disease adopting MP-derived metrics as primary [96, 97], secondary [79, 98, 146-149], or exploratory endpoints $[150,151]$. In 1 case the endpoint type was not clear [152]. Of particular note, 2 mul- ticentre international randomised controlled trials (RCTs) successfully employed MAIA MP. The Macular Telangiectasia Type 2-Phase 2 CNTF Research Group recruited across 11 sites, collecting secondary endpoint data, whilst the LEAD Study Group recruited at 6 sites, collecting exploratory endpoint data.

Interventions and conditions assessed by MP endpoints include lutein supplementation $[79,146]$ and oral telomerase for early AMD [96], subthreshold micropulse laser in intermediate AMD [150], photodynamic therapy (PDT) treatment regimens for age-related $[97,147]$ and myopic CNV [151], intravitreal treatments for neovascular AMD [148], suprachoroidal cell autograft [152], and lampalizumab [98] for GA and a CNTF (ciliary neurotrophic factor) implant in macular telangiectasia [149].

To date, only mesopic MP has been included as a trial endpoint. Whilst earlier trials employed the MP-1, more recent studies have opted for the MAIA device. MS was the most commonly reported outcome. A range of other raw data-defined metrics have been used, either modifications on MS or a surrogate for scotoma size.

Employed as a secondary endpoint, Huang et al. [79] described MS values over the central 1,3 , and $5^{\circ}$ in an RCT investigating potential functional benefits of lutein supplements. Those taking either 10 or $20 \mathrm{mg}$ of lutein had a greater increase in foveal sensitivity over $1^{\circ}$ compared to a placebo group, an effect that was not evident when overall MS was considered.

In a multicentre RCT examining the effect of CNTF on retinal neurodegeneration in macular telangiectasia, aggregate sensitivity loss was reported as a post hoc analysis [149]. Aggregate sensitivity loss considers both structure and function and relies on superimposition of SD-OCT and MP data. The technique was first described by Sallo et al. [153] using MP-1 data, and subsequently MAIA data [154]. Briefly, the inner segment/outer segment (IS/OS) break is defined on SD-OCT. Considering only stimuli within the central $10^{\circ}$ of the grid, sensitivity values outside the IS/OS break are averaged and termed the background sensitivity. Individual sensitivity values of points falling within the IS/ OS break are subtracted from the background sensitivity value. These differences are then summed and deducted from the background sensitivity value to give the aggregate sensitivity loss in $\mathrm{dB}$. As such, aggregate sensitivity is a volumetric measure of scotoma depth and an example of a condition-specific, researcher-driven metric.

Other scotoma-based outcomes have also been used in relation to macular disease, such as the percentage or number of reduced, relative, or absolute scotomatous points [96-98]. In general, these metrics displayed concordance 
with the MS measures also reported in the individual studies. Further developing this theme, a longitudinal observational study of early and intermediate AMD plus normal controls compared the ability of several visual function outcomes to track progression over a 12-month period [145]. In addition to MS, the percentage reduced threshold (PRT), expressed as the percentage of points falling below $25 \mathrm{~dB}$ on MAIA testing, was deduced. PRT was purported to be the most sensitive measure to map progression as it declined significantly in all 3 groups over 6 and 12 months. The utility of MS of perilesional points and PRT as interventional trial endpoints have yet to be tested.

Though strictly falling outside the parameters of our search, novel potential treatments for GA have been assessed in phase I/II and II studies, including neuroprotective agents, visual cycle inhibitors, immune modulating agents, and antioxidants [155]. Frequently, these trials have not included MP endpoints, presumably due to their early stage. However, the MP-1 was included as a secondary outcome in open label trials examining the safety and efficacy of the topically administered antioxidant OT-551 [88], and the immunosuppressive agent Sirolimus delivered subconjunctivally [89] and intravitreally [90]. Rather than report the MS of all points examined, the average of all non-scotomatous points (defined at baseline) was calculated, thus minimising the floor effects of non-seeing retina. Ultimately efficacy of these treatments was not established, but these studies indicate MP has a place in future interventional trials in GA.

Additionally, utilising MP data of those receiving OT-551 topically, additional GA-specific metrics were outlined [156]. Intended to track the progression of atrophy, Meleth et al. [156] evaluated both the number of scotomatous points (no response to brightest stimuli) and the MS of perilesional points (points immediately adjoining a scotomatous point). Significant per year progression was evident in each measure $(+4.4$ points and $-1.20 \mathrm{~dB}$, respectively, $p<0.004)$, suggesting promise as endpoints in future trials. In a similar vein, a novel deep scotoma mapping strategy using the physiological blind spot has been conceptualised in normal eyes [157]. Using 2 grids, the second with more points tightly spaced and centred on the optic nerve head, Wu et al. [157] simulated scotoma progression. Their deep scotoma mapping strategy of probing the optic nerve head with single $10-\mathrm{dB}$ stimuli resulted in an almost 2 -fold increase in the ability to detect simulated progression versus a standard 4-2 staircase approach. It was anecdotally more agreeable to subjects. Although additional validation is needed, deep scotoma mapping could im-

Microperimetry: Trial Endpoint in Retinal Disease prove the accuracy of tracking progression in atrophic retinal changes.

With respect to future alternative endpoints, reporting change in PWS is also likely to be important. PWS offers a more robust way of identifying local alterations and, in combination with multimodal imaging, has enhanced our understanding of specific functional deficits present with precise structural changes in AMD [55, 143, 144]. In fact, PWS over reticular pseudodrusen has been shown to exhibit faster progression than that detected in unremarkable retinal regions in the same eye. This effect was observed under mesopic and scotopic conditions using the MP-1S [144]. Similar analysis in eyes with large drusen demonstrated the same effect under the mesopic condition only [143]. Scotopic data in both studies were censored to some extent with the exclusion of participants who required a change of ND filter throughout the 3-year follow-up period. On the basis that they required a filter change, these eyes may be the ones experiencing the most change. Future studies on the MP-3S or S-MAIA, devices that do not rely on manipulation of ND filters and also have large dynamic ranges, will further enhance our understanding of longitudinal change in scotopic function in AMD.

The granularity with which we can functionally interrogate retinal lesions MP exposes some of the frailties of structurally defining disease severity. Pfau et al. [55], using mesopic and 2-colour dark-adapted MP, demonstrated discrete functional phenotypes in eyes with cuticular, reticular, and soft drusen which would all be classified as having intermediate AMD. Similarly, Hsu et al. [145] demonstrated longitudinal functional decline in MP measures despite no change in disease severity classification.

\section{Diabetic Macular Oedema}

Many landmark DMO treatment trials over the last decade have not included MP endpoints, preferring BCVA and structural outcomes [158-163]. Nevertheless, we identified 5 randomised studies of laser and/or intravitreal drug treatments for DMO listing MP as a primary $[80,81]$, secondary $[164,165]$, or exploratory [82] endpoint, each using the MP-1 device. Where MP has been included as an endpoint to date, there is general consistency in device, test strategies, and metrics, allowing for potentially easier cross-trial comparison.

Vujosevic et al. [80] defined MS and foveal MS (FMS) over central $4^{\circ}$ as primary endpoints in a single centre trial comparing ETDRS laser photocoagulation to subthreshold micropulse diode laser in DMO (SMDL). Sig- 
nificant improvements in MS and $4^{\circ}$ FMS were observed only in those treated with SMDL, and the change between groups for both metrics was significant. In a later study of yellow versus infrared SMDL by the same group [81] using the same endpoints, no change in MS and $4^{\circ} \mathrm{FMS}$ was shown. However, stratifying results by baseline MS showed those whose baseline MS fell between 15 and 18 $\mathrm{dB}$ had significant within-group improvements in MS, and $4^{\circ}$ FMS in both yellow and infrared SMDL groups. The limited dynamic range of the MP-1 leaves MS susceptible to ceiling effects. Stratification by baseline value mitigates this, particularly when baseline values are high [81].

LUCIDATE, a single-centre RCT, also adopted MS and $4^{\circ} \mathrm{FMS}$, but as an exploratory endpoint in a study of ranibizumab versus ETDRS laser in DMO [82]. A subgroup of the Da Vinci study cohort, a multicentre RCT comparing doses and dosing regimens of intravitreal aflibercept to ETDRS laser [166], were examined by MP-1 to assess treatment-related changes in MS [164]. A customised grid aligned with OCT subfields was used. MS was calculated in the central $4^{\circ}$, inner $10^{\circ}$, and inner to outer ring $\left(2-8^{\circ}\right.$ radius).

The Diabetic Retinopathy Research Group Vienna recently published MP-1 results [165] from a single-centre prospective randomised study of bevacizumab versus triamcinolone for DMO [167]. Presented as a secondary outcome in a standalone report, MP variables were defined as MS, absolute scotoma size ( $\%$ of absolute $[<0 \mathrm{~dB}]$ scotoma points), and relative scotoma size ( $\%$ of relative $[\geq 1 \mathrm{~dB}$ and $<10 \mathrm{~dB}$ ] scotoma points). MS significantly improved in bevacizumab-treated eyes, mirrored by significant reductions in absolute and relative scotoma size.

As efforts to find new therapies in DMO continue, early-phase trials have included MS metrics as secondary endpoints, notably in a phase I/II trial of oral dextromethorphan [168] and a phase II trial of cibinetide [169]. We are unaware of test-retest values derived from DMO cohorts which may hamper understanding of the minimal change thought to be clinically significant. Undertaking this preparatory work could help define the value of $\mathrm{MP}$ as an endpoint in DMO, particularly given its inclusion in these recent early-phase studies.

Given that mesopic retinal sensitivity deficits have been identified in diabetes prior to the development of diabetic retinopathy [170], MP may be a candidate endpoint as treatments are developed for earlier disease. In fact, MAIA-derived mesopic MS and macular integrity index were defined as primary endpoints in a non-randomised prospective controlled study of docosahexaenoic acid supplementation in non-proliferative diabetic ret- inopathy [171]. Although mesopic measures may hold promise, scotopic MP has not identified rod-based functional deficits in diabetic eyes with or without non-proliferative diabetic retinopathy [172] and so focus will likely remain on mesopic measures.

\section{Central Serous Chorioretinopathy}

Three randomised studies of CSCR treatments were identified, each reporting a structural primary endpoint and mesopic MS as a secondary endpoint [173-175]. No other MP metrics were reported.

The efficacy of half-dose PDT over high-density subthreshold micropulse laser (HSML) in chronic CSCR was established in a large multicentre RCT, the PLACE trial, undertaken at 5 academic medical centres across Europe [173]. The primary outcome was resolution of subretinal fluid (SRF), whilst secondary functional endpoints were functional (BCVA and MS) [176]. SRF resolved in significantly more eyes receiving half-dose PDT than HSML. Concordant changes in visual function were demonstrated, with half-dose PDT patients showing a significantly higher increase in BCVA and MS.

Data on a subgroup of PLACE subjects with persistent SRF at study conclusion were recently published in the very aptly named REPLACE crossover trial [174]. Crossover to the half-dose PDT group showed a significant improvement in MS, without improvement in BCVA.

Successful use of MP in a large international multicentre trial setting is significant. However, MP testing strategies were not specified beyond acknowledging that examinations were performed on 2 devices (MP-1 and MAIA), with subjects followed up on the same device. Measurement scales were aligned using a conversion method described by Parodi et al. [63] in a small cross-sectional pilot study of eyes with IRD and normal controls.

\section{Vitreoretinal Surgery}

Seven randomised studies of vitreomacular surgery outcomes and techniques were identified using mesopic MP measures as primary $[86,87]$ or secondary $[83-85,177,178]$ endpoints. MP-1, MAIA, and OCT/SLO devices were used. In addition to MS, measures of foveal function over the central $2[86]$ and $4^{\circ}[83-85,87]$ and number of absolute scotoma points [87] have been defined as outcome measures in randomised vitreoretinal surgery studies. Of particular note, a multicentre RCT comparing the merits of ILM peel during vitrectomy for idiopathic macular pucker using $4^{\circ}$ FMS as a primary endpoint, revealed significantly better fo- 
veal function in eyes without ILM peel, despite no difference in BCVA between treatment groups [87].

FMS over $4^{\circ}$ has also been used as a secondary endpoint in single-centre randomised studies comparing outcomes of complete versus foveal sparing ILM peels in both macular hole surgery $[83,84]$ and epiretinal membrane removal [85]. Change in BCVA, the primary outcome in these 3 studies, was not significantly different between groups, whereas change in $4^{\circ} \mathrm{FMS}$ was significantly higher in the foveal sparing arms of all 3 studies. Although FMS has been shown to have a significant moderate correlation to BCVA in eyes undergoing vitreomacular surgery [86], these results suggest FMS may be better able to describe changes in foveal function following vitreomacular surgery than BCVA alone.

\section{Conclusion}

While MP is yet to be fully established as a clinical trial endpoint, undoubtedly there is abundant interest in its utility as such, underlined by the scope of its uptake demonstrated in this review, including in endpoint development studies $[8,12,13,15]$ as well as the seemingly countless ways in which novel metrics from raw data are being conceived. Where MP has been taken up as an endpoint in both inherited and acquired retinal disease, it has predominantly been used as a secondary outcome to date. BCVA persists as the main functional outcome measure of choice; however, we did see notable exceptions in AMD $[96,97]$, macular oedema $[80,81,179]$, and even vitreoretinal surgery $[86,87]$.

In addition to interest in and uptake of MP, this review illustrates the extensive variation in how it is being employed in terms of device, test strategy, and reported metrics. Given the breadth and heterogeneity of retinal conditions, it would be unrealistic to expect one optimal test strategy or all-encompassing metric. More credible is the concept of condition or treatment-specific approaches. The custom features of MP devices provide a fertile environment for this. As illustrated in Table 3, examples of MP features being used in this way are frequently seen, be that via grid customisation in choroideremia or surgical procedures, or exploiting raw data to create conditiontargeted indices as in GA and Stargardt disease.

Whilst tailoring an exam to a treatment area, lesion, or expected drug effect is desirable, clinical trial endpoints are by their very nature required to be standardised. It is of course possible to standardise what was once custom; however, without more overlap in strategies and report-

Microperimetry: Trial Endpoint in Retinal Disease ing, it may be difficult to accumulate a sufficient body of evidence to validate a particular strategy. The current lack of consensus may be stifling the development of well-defined MP endpoints and the opportunity to compare results across trials. Transparent and detailed reporting of test strategies, especially where customisation is relied upon and novel metrics used, is a must.

Endpoint development for MP is still in its infancy; however, achievements in SAP glaucoma analysis may guide its next steps. There have already been explicit attempts to replicate SAP visual field indices in retinal conditions using MP data. Pattern deviation, total deviation, mean defect, mean deviation, pattern standard deviation, and loss variance have been evaluated in recent studies $[76,180]$. Cluster analysis has also been used to describe disease-specific patterns of visual field defects [180]. Although intuitive in glaucoma, this type of functional grouping may not be so readily achieved for retinal disease given its heterogeneity. It remains to be seen which indices may be adopted and for which retinal diseases. What would be of great practical benefit is the development of software that performs automated statistical analysis, like the GCP (Glaucoma Change Probability) software, which compares pointwise changes with an averaged baseline (often from 2 or 3 tests) and flags up changes that exceed the expected variability [181]. The robust establishment of normative data, together with TRTV data, as discussed later, are essential prerequisites for this.

To our best knowledge, regulatory authorities currently do not recognise any MP metrics as clinical trial endpoints. Yet again, the example of SAP may provide insight into what regulators may reasonably expect. For instance, the FDA and National Eye Institute Glaucoma Clinical Trial Design and Endpoints Symposium suggested that visual field progression may be an adequate primary endpoint, specifically: a between-group difference in visual field progression with 5 or more points showing significant changes from baseline or a statistically and clinically significant between-group difference across the total visual field, purported to be $7 \mathrm{~dB}$ [182]. Therefore, we anticipate that any MP endpoint metrics will involve stipulations on the number of points demonstrating change and an established threshold sensitivity value to cross, presumably according to the disease and device used.

A discussion of endpoints would not be complete without consideration of the practical elements of implementing the said endpoint in a multicentre clinical trial setting. No amount of repeatability or sensitivity can confirm the value of an endpoint if it is impractical to measure. MP has a reputation of being a lengthy, burdensome 
test for patients and operators alike. Even as devices have become more automated and test durations shorter, this perception has persisted $[183,184]$, and a recent study cited patient refusal to complete MP as limiting longitudinal data collection [145]. Although high-quality MP data can certainly be obtained in laboratory and small clinical study settings by motivated researchers and clinicians, it remains to be seen whether this can be scaled up appropriately, but there are positive signs.

The LEAD study, though designating MP as an exploratory endpoint, should be commended for successfully co-ordinating MAIA data collection for 280 subjects with intermediate AMD at 5 Australian and 1 Northern Irish site in an interventional RCT - a very significant achievement. Adding further weight to the viability of MP in large-scale trials, the natural history study ProgStar recently published 12-month follow-up MP-1 data on 359 eyes with Stargardt disease from 9 sites across the USA and Europe [185], a very meaningful accomplishment given the significant visual impairment of this cohort.

That being said, pivotal trials for anti-vascular endothelial growth factor in neovascular AMD [3-5] and DMO $[158,160]$ recruited at $70-150$ sites across international borders. Even if, as hoped, more sensitive validated endpoints make smaller, faster trials a reality, it is still exceedingly likely that trials for novel treatments in high prevalence conditions such as AMD or DMO will be conducted at a large number of sites. So far, it has not been shown whether a large, high-quality MP data set can be acquired under such circumstances. Each with 20 international sites, MACUSTAR [12] and the AMD Ryan Initiative Study (ARIS) [14] will offer further insight into this within the context of AMD. In contrast, gene therapy trials typically recruit at a small number of specialist tertiary centres, potentially making practical considerations somewhat easier to manage.

MP requires trained, skilled operators, with each site needing at least 2 personnel depending on the size of the trial. Operators should be certified as being able to perform the test to the required standard. This is usually assessed by adherence to a standard operating procedure, reliability indices, and image quality of a set number of examinations on normal eyes and eyes with the pathology under investigation. Clinical experience has shown us that engagement with operators and clinical sites, especially if new to the technique, is essential. A proficient, confident operator stands the best chance of capturing accurate data and making the examination acceptable to patients. After all, a primary endpoint assessment that too few patients can complete is not viable.
Although important, knowing how to technically operate the instrument is not the only consideration. Patient instruction needs to be clear, concise, and consistent. Whilst newer devices are more automated, operators need to keep patients engaged and focused throughout the examination, remaining reactive to signs that compliance is waning, such as wandering fixation or closing eyelids. Positive, constructive, and ongoing feedback is key. Regular data quality reviews should be implemented and feedback provided to operators and sites wherever protocol deviations or missing data are observed and of equal importance, when data quality is high.

Endorsement of scotopic MP as an endpoint brings added challenges. A period of at least $30 \mathrm{~min}$ of DA is a prerequisite for scotopic testing, requiring patients to sit in light-tight, dark room conditions. This needs a windowless room, with a light-tight seal around the door and any artificial light sources (e.g., computer screens, power light, exit signs) within the room need to be disabled or covered by a long wavelength red filter. It is important to emphasise the distinction between these conditions and, for instance, a cubicle with dimmed light adequate for SAP. Many clinical trial centres will not have ready access to suitable dark room conditions. Prior planning and organisation will likely be necessary, as well as some form of monitoring to ensure appropriate conditions are achieved and maintained for the duration of the study. From commencement of DA to the completion of testing, the dark room conditions must be preserved. If light enters the room during testing, the data collected will not be valid. This, combined with the specificity of the conditions, dictates that a dedicated, sole-purpose room is desirable.

The experience of total darkness can be unpleasant. This impacts on patients and operators alike, who will both be required to remain within these conditions for the entire DA and testing period. Again, the skill and reassurance of the operator will be key in ensuring these circumstances can be tolerated by patients. Adherence to a full period of DA is mandatory for data validity. A means of ensuring adherence across all trial sites should be implemented. Though not insurmountable, implementation of scotopic testing on a large scale certainly has test-specific challenges to manage.

Despite there being no examples of scotopic MP being employed as a primary or secondary endpoint in an interventional clinical trial so far, it is very encouraging that the SMART study, which scrutinises the potential role of scotopic MP as an endpoint in Stargardt disease, has recruited 118 participants [57]. Furthermore, scotopic MP has also been included in endpoint development studies in AMD [12-14]. 
Adopting MP as a primary endpoint on a large multicentre clinical trial will also impact the budget. Commercially available microperimeters are expensive and a clinical reading centre will be necessary to provide standardised, objective, anonymised grading of results [186]. Arguably, costs may be similar to those incurred when employing imaging modalities such as OCT. However, should MP replace existing functional assessments which require minimal equipment and no external grading such as BCVA, the cost differential is likely to be substantial.

Given the choice of commercially available devices, the decision of which instrument to use in a trial also requires considerable thought. It is certainly not desirable to use more than 1 device during a trial given the incompatibility of $\mathrm{dB}$ scales across devices. Therefore, a high level of upfront commitment is necessary. Deliberation should include whether analysis will require comparison with a normative database, which stimuli and grid settings may be most appropriate, under which luminance conditions retinal sensitivity should be measured, and how the patient population of interest will fare on a given device or test strategy. That said, researchers may deliberatively want to match test settings and pre-test DA protocols to allow direct comparison across interventions. In addition to new instruments becoming available, software and hardware updates are periodically released, bringing in new features and phasing out others. The impact of such updates on trial data collection should therefore be established before implementing any changes.

Yet further still, during the course of a clinical study, the pros and cons of particular devices and test strategies may become apparent. If implementing MP in a patient population for the first time, pilot testing is advisable. Instrument costs likely prohibit piloting different devices, but the option of trialling differing test strategies is feasible. This also allows for determination of TRTV in the patient population under investigation, an approach previously used in AMD [187], XLRS [188], and RPGR retinitis pigmentosa [189]. Intuitively it is expected that TRTV may vary with baseline retinal sensitivity. Although this effect has been observed [99], so too has the independence of baseline sensitivity from TRTV [190].

The results of TRTV studies across a breadth of retinal disease introduce further considerations for trialists. CoR is often presented as a threshold change that is clinically meaningful as a smaller change may be considered measurement error [99, 188, 189, 191]. However, caution has been urged in defining treatment response related to TRTV variability without taking inter-examiner effects into account [78] as it is highly likely that multiple operators may

Microperimetry: Trial Endpoint in Retinal Disease perform MP assessments over the course of a clinical trial. Furthermore, if TRTV is defined in eyes with a pre-existing dense scotoma, PWS CoR may be inflated due to the increased variability of PWS on the scotoma edge [77]. In this case, the use of PWS CoR as the threshold for clinically significant change may be setting the bar too high.

Whether TRTV is assessed with or without the followup mode enabled will likely impact on CoR. The use of a follow-up mode ensures the same retinal locations are examined on retesting, which in dedicated endpoint-exploring studies is ideal. However, patients enrolled in interventional trials often perform repeat testing as part of their baseline assessment. If the follow-up mode was enabled in these cases, this would result in the selection of a pre-intervention test as follow-up. To avoid this, researchers may decide to perform pre-intervention baseline assessments without the use of the follow-up mode, but this is likely to result in higher TRTV values being obtained. Additionally, in follow-up mode, the starting stimulus intensity at any given point is informed by the values obtained in the baseline test (either at or near the baseline value), thus contributing to a shorter examination duration [52].

The presence of learning effects in MP has also been explored extensively within TRTV studies, the results of which have implications for clinical trial design. Learning effects have been confirmed in those without prior experience of MP, culminating in improved performance on a repeat test $[74,99]$, with authors advocating that the first examination be considered practice only. Conversely, in other studies learning effects have not been observed, although a truncated practice examination was performed prior to testing in these cases $[59,191]$. Despite such disparities, it is recommended MP protocols include some form of practice session or exam before baseline testing.

Given that some gene therapies may target IRDs in children, it is encouraging that the viability of MAIA testing in children with normal vision between the ages of 9 and 12 years has been reported [192]. However, in comparison to adults with normal vision, CoR was significantly higher and averaging of multiple tests was advised. Further work to establish TRTV limits in children with IRD is warranted.

In addition to considering retinal sensitivity in isolation, the utility of composite endpoints incorporating MP has also been raised. A composite endpoint generally comprises multiple single independent endpoints which on their own may not possess sufficient reliability or sensitivity, but do so in combination [193]. Using SAP, a combined structure and function index has been shown to perform better than isolated measures in glaucoma detection and staging 
[194]. Indeed, the diagnostic ability of such an index performs better in eyes with field loss when MP versus SAP is used [195]. A similar structure-function approach has been suggested for future $A B C A 4$ trials [196] and composite approaches incorporating MP have been proposed in CSCR [197], AMD [198], and IRD generally [132].

Moreover, the potential of numerous OCT-defined structural indices to act as surrogate biomarkers for retinal sensitivity have also been reported, specifically in AMD, DMO, macular telangiectasia, and Stargardt disease. Across this spectrum, ellipsoid zone loss/integrity, retinal pigment epithelium drusen complex, hyper-reflective loci, outer-retinal thickness, reticular pseudodrusen, nascent GA, and pigment clumping have all shown promise as retinal sensitivity biomarkers $[8,144,199$ 204]. Of course, surrogate structural endpoints will only be of interest if shown to be associated with visual function loss [1]. Furthermore, artificial intelligence has brought exciting innovation to this field. Deep learning models have been developed that can reliably predict or "infer" mesopic and scotopic retinal sensitivity based on imaging data alone in AMD [183] and macular telangiectasia [184]. Although further validation is necessary, these are exciting new avenues to explore.

If, as we all hope, novel interventions for retinal disease are established, recipients of such therapies will need to be monitored and assessed for treatment response in routine clinical practice. Indeed, one of the great successes of SAP has been its crossover to routine clinical use; it is almost universally available, frequently repeated in patients, and familiar to clinicians. The same cannot be said of MP currently, and even with time and ensuing familiarity, such practical considerations, like the ones we have described, may impact its crossover from research to clinical practice. However, if structural biomarkers and/or AI-derived pseudo-functional outcomes were to be validated, hypothetically a single objective OCT scan could replace mesopic and scotopic MP examinations in the future. This has the potential to transform clinical trial design, reducing patient burden, equipment costs and, via frequent, early data capture, study durations.

In conclusion, despite the current lack of consensus, there are encouraging signs that MP may deliver on the promise of endpoint validity. Endpoint development trials will undoubtedly be key in understanding the validity of MP as a clinical trial endpoint, but existing signs are promising.

\section{Acknowledgements}

We would like to gratefully acknowledge (in alphabetical order): Mauro Campigotto (NIDEK Technologies, Padova, Italy), Marco Morales (former co-founder of CenterVue, Padova, Italy), and Carlo Pellizzari (CenterVue, Padova, Italy) for their technical advice and clarifications.

\section{Conflict of Interest Statement}

Y.Y.: none. H.D.: Boehringer-Ingelheim.

\section{Funding Sources}

Y.Y.: None. H.D.: time funded from the Innovative Medicines Initiative 2 Joint Undertaking 439 under grant agreement No. 116076. This Joint Undertaking receives support from the $440 \mathrm{Eu}$ ropean Union's Horizon 2020 research and innovation programme and EFPIA.

\section{Author Contributions}

Y.Y. and H.D.: conceptualisation, methodology, analysis, writing - original draft, review, and editing.

\section{References}

1 Csaky KG, Richman EA, Ferris FL 3rd. Report from the NEI/FDA ophthalmic clinical trial design and endpoints symposium. Invest Ophthalmol Vis Sci. 2008 Feb;49(2): $479-89$.

2 Ferris FL 3rd, Podgor MJ, Davis MD, Group DR. Macular edema in diabetic retinopathy study patients: diabetic retinopathy study report number 12. Ophthalmology. 1987 Jul; 94(7):754-60.

3 Brown DM, Michels M, Kaiser PK, Heier JS, Sy JP, Ianchulev T. Ranibizumab versus verte- porfin photodynamic therapy for neovascular age-related macular degeneration: two-year results of the ANCHOR study. Ophthalmology. 2009 Jan;116(1):57-65.e5.

4 Rosenfeld PJ, Brown DM, Heier JS, Boyer DS, Kaiser PK, Chung CY, et al.; MARINA Study Group. Ranibizumab for neovascular age-related macular degeneration. $\mathrm{N}$ Engl J Med. 2006 Oct;355(14):1419-31.

5 Heier JS, Brown DM, Chong V, Korobelnik JF, Kaiser PK, Nguyen QD, et al.; VIEW 1 and VIEW 2 Study Groups. Intravitreal afliber- cept (VEGF trap-eye) in wet age-related macular degeneration. Ophthalmology. 2012 Dec; 119(12):2537-48.

6 Lesmes LA, Jackson ML, Bex P. Visual function endpoints to enable dry AMD clinical trials. Drug Discov Today Ther Strateg. 2013; 10(1):e43-50.

7 Dimopoulos IS, Tseng C, MacDonald IM. Microperimetry as an outcome measure in choroideremia trials: reproducibility and beyond. Invest Ophthalmol Vis Sci. 2016 Aug;57(10): 4151-61. 
8 Strauss RW, Ho A, Muñoz B, Cideciyan AV, Sahel JA, Sunness JS, et al.; Progression of Stargardt Disease Study Group. The Natural History of the Progression of Atrophy Secondary to Stargardt Disease (ProgStar) Studies: Design and Baseline Characteristics: ProgStar Report No. 1. Ophthalmology. 2016 Apr;123(4):817-28.

9 Nair P, Aiello LP, Gardner TW, Jampol LM, Ferris FL 3rd. Report from the NEI/FDA diabetic retinopathy clinical trial design and endpoints workshop. Invest Ophthalmol Vis Sci. 2016 Oct;57(13):5127-42.

10 Csaky K, Ferris F 3rd, Chew EY, Nair P, Cheetham JK, Duncan JL. Report from the NEI/FDA endpoints workshop on age- related macular degeneration and inherited retinal diseases. Invest Ophthalmol Vis Sci. 2017 Jul; 58(9):3456-63.

11 Thompson DA, Iannaccone A, Ali RR, Arshavsky VY, Audo I, Bainbridge JW, et al.; Monaciano Consortium. Advancing Clinical Trials for Inherited Retinal Diseases: Recommendations from the Second Monaciano Symposium. Transl Vis Sci Technol. 2020 Jun;9(7):2-2.

12 Finger RP, Schmitz-Valckenberg S, Schmid M, Rubin GS, Dunbar H, Tufail A, et al.; on behalf of the MACUSTAR consortium. MACUSTAR: development and clinical validation of functional, structural, and patientreported endpoints in intermediate age-related macular degeneration. Ophthalmologica. 2019;241(2):61-72.

13 Curcio CA, McGwin G Jr, Sadda SR, Hu Z, Clark ME, Sloan KR, et al. Functionally validated imaging endpoints in the Alabama study on early age-related macular degeneration 2 (ALSTAR2): design and methods. BMC Ophthalmol. 2020 May;20(1):196.

14 Wright C, Mazzucco AE, Becker SM, Sieving PA, Tumminia SJ. NEI-supported age-related macular degeneration research: past, present, and future. Transl Vis Sci Technol. 2020 Jun; 9(7):49-49.

15 Iftikhar M, Usmani B, Sanyal A, Kherani S, Sodhi S, Bagheri S, et al. Progression of retinitis pigmentosa on multimodal imaging: the PREP-1 study. Clin Exp Ophthalmol. 2019 Jul;47(5):605-13.

16 Meinert CL. Clinical trials, design, conduct, and analysis. New York: Oxford University Press; 1986.

17 US National Library of Medicine. Clinicaltrials.gov. [cited 09/09/2020]; Available from: https://clinicaltrials.gov/ct2/results?term $=\mathrm{mi}$ croperimetry\&pg $=4$.

18 Fujii GY, De Juan E Jr, Humayun MS, Sunness JS, Chang TS, Rossi JV. Characteristics of visual loss by scanning laser ophthalmoscope microperimetry in eyes with subfoveal choroidal neovascularization secondary to agerelated macular degeneration. Am J Ophthalmol. 2003 Dec;136(6):1067-78.

19 Yodoi Y, Tsujikawa A, Nakanishi H, Otani A, Tamura H, Ojima Y, et al. Central retinal sensitivity after intravitreal injection of bevaci- zumab for myopic choroidal neovascularization. Am J Ophthalmol. 2009 May; 147(5): 816-24, 824.e1.

20 Vujosevic S, Midena E, Pilotto E, Radin PP, Chiesa L, Cavarzeran F. Diabetic macular edema: correlation between microperimetry and optical coherence tomography findings. Invest Ophthalmol Vis Sci. 2006 Jul;47(7): 3044-51.

21 Roesel M, Heimes B, Heinz C, Henschel A, Spital G, Heiligenhaus A. Comparison of retinal thickness and fundus-related microperimetry with visual acuity in uveitic macular oedema. Acta Ophthalmol. 2011 Sep;89(6): 533-7.

22 Sugiura A, Fujino R, Takemiya N, Shimizu K, Matsuura M, Murata $\mathrm{H}$, et al. The association between visual function and retinal structure in chronic central serous chorioretinopathy. Sci Rep. 2017 Nov;7(1):16288.

23 Fujino R, Asaoka R, Aoki S, Sugiura A, Kusakabe M, Asano-Shimizu K, et al. The usefulness of the retinal sensitivity measurement with a microperimetry for predicting the visual prognosis of branch retinal vein occlusion with macular edema. Graefes Arch Clin Exp Ophthalmol. 2020 Sep;258(9):1949-58.

24 Giuliari GP, Pujari S, Shaikh M, Marvell D, Foster CS. Microperimetry findings in patients with birdshot chorioretinopathy. Can J Ophthalmol. 2010 Aug;45(4):399-403.

25 Amari F, Ohta K, Kojima H, Yoshimura N. Predicting visual outcome after macular hole surgery using scanning laser ophthalmoscope microperimetry. Br J Ophthalmol. 2001 Jan; 85(1):96-8.

26 Dal Vecchio M, Lavia C, Nassisi M, Grignolo FM, Fea AM. Microperimetric assessment after epiretinal membrane surgery: 4-year follow-up. J Ophthalmol. 2016;2016:7030791.

27 Tanna P, Georgiou M, Aboshiha J, Strauss RW, Kumaran N, Kalitzeos A, et al. Crosssectional and longitudinal assessment of retinal sensitivity in patients with childhood-onset stargardt disease. Transl Vis Sci Technol. 2018 Nov;7(6): 10.

28 Xue K, Jolly JK, Barnard AR, Rudenko A, Salvetti AP, Patrício MI, et al. Beneficial effects on vision in patients undergoing retinal gene therapy for choroideremia. Nat Med. 2018 Oct;24(10):1507-12.

29 Dimopoulos IS, Hoang SC, Radziwon A, Binczyk NM, Seabra MC, MacLaren RE, et al. Two-Year Results After AAV2-Mediated Gene Therapy for Choroideremia: the Alberta Experience. Am J Ophthalmol. 2018 Sep;193: $130-42$.

30 Lam BL, Davis JL, Gregori NZ, MacLaren RE, Girach A, Verriotto JD, et al. Choroideremia Gene Therapy Phase 2 Clinical Trial: 24-Month Results. Am J Ophthalmol. 2019 Jan; 197:65-73.

31 Cukras C, Wiley HE, Jeffrey BG, Sen HN, Turriff A, Zeng Y, et al. Retinal AAV8-RS1 gene therapy for X-linked retinoschisis: initial findings from a phase I/IIa trial by intravitreal delivery. Mol Ther. 2018 Sep;26(9):2282-94.
32 Cehajic-Kapetanovic J, Xue K, Martinez-Fernandez de la Camara C, Nanda A, Davies A, Wood LJ, et al. Initial results from a first-inhuman gene therapy trial on X-linked retinitis pigmentosa caused by mutations in RPGR. Nat Med. 2020 Mar;26(3):354-9.

33 Hanout M, Horan N, Do DV. Introduction to microperimetry and its use in analysis of geographic atrophy in age-related macular degeneration. Curr Opin Ophthalmol. 2015 May;26(3):149-56.

34 Jean B, Frohn A, Thiel H. Laser scanning in ophthalmology. Fortschritte der Ophthalmologie. Zeitschrift der Deutschen Ophthalmologischen Gesellschaft. 1990;87(2):158.

35 Timberlake GT, Mainster MA, Peli E, Augliere RA, Essock EA, Arend LE. Reading with a macular scotoma. I. Retinal location of scotoma and fixation area. Invest Ophthalmol Vis Sci. 1986 Jul;27(7):1137-47.

36 Crossland M, Jackson ML, Seiple WH. Microperimetry: a review of fundus related perimetry. Optometry Reports. 2012;2(1):e2.

37 Midena E, Vujosevic S, Cavarzeran F, Microperimetry G. Study, Normal values for fundus perimetry with the microperimeter MP1. Ophthalmology. 2010 Aug;117(8):1571-6, 1576.e1.

38 Liu H, Bittencourt MG, Wang J, Sophie R, Annam R, Ibrahim MA, et al. Assessment of central retinal sensitivity employing two types of microperimetry devices. Transl Vis Sci Technol. 2014 Sep;3(5):3-3.

39 Nguyen CT, Fraser RG, Tan R, Caruso E, Lek JJ, Guymer RH, et al. Longitudinal changes in retinotopic rod function in intermediate agerelated macular degeneration. Invest Ophthalmol Vis Sci. 2018 Mar;59(4):AMD19-24.

40 Schmitz-Valckenberg S, Fan K, Nugent A, Rubin GS, Peto T, Tufail A, et al. Correlation of functional impairment and morphological alterations in patients with group $2 \mathrm{~A}$ idiopathic juxtafoveal retinal telangiectasia. Arch Ophthalmol. 2008 Mar;126(3):330-5.

41 Wågström J, Larsen M. Scotopic and photopic dissociation in patients with chronic central serous chorioretinopathy. Invest Ophthalmol Vis Sci. 2014;55(13):5872-5872.

42 Kabanarou SA, Holder GE, Fitzke FW, Bird AC, Webster AR. Congenital stationary night blindness and a "Schubert-Bornschein" type electrophysiology in a family with dominant inheritance. Br J Ophthalmol. 2004 Aug; 88(8):1018-22.

43 Dias MF, Joo K, Kemp JA, Fialho SL, da Silva Cunha A Jr, Woo SJ, et al. Molecular genetics and emerging therapies for retinitis pigmentosa: basic research and clinical perspectives. Prog Retin Eye Res. 2018 Mar;63:107-31.

44 Miyake Y, Shinoda K. Clinical Electrophysiology. RETINA. 5th ed. ed. Amsterdam: Elsevier Mosby; 2012.

45 Jacobson SG, Voigt WJ, Parel JM, Apáthy PP, Nghiem-Phu L, Myers SW, et al. Automated light- and dark-adapted perimetry for evaluating retinitis pigmentosa. Ophthalmology. 1986 Dec;93(12):1604-11. 
46 Bennett LD, Klein M, Locke KG, Kiser K, Birch DG. Dark-adapted chromatic perimetry for measuring rod visual fields in patients with retinitis pigmentosa. Transl Vis Sci Technol. 2017 Aug;6(4):15-15.

47 Flamendorf J, Agrón E, Wong WT, Thompson D, Wiley HE, Doss EL, et al. Impairments in dark adaptation are associated with agerelated macular degeneration severity and reticular pseudodrusen. Ophthalmology. 2015 Oct;122(10):2053-62.

48 Curcio CA, Medeiros NE, Millican CL. Photoreceptor loss in age-related macular degeneration. Invest Ophthalmol Vis Sci. 1996 Jun; 37(7):1236-49.

49 Nebbioso M, Barbato A, Pescosolido N. Scotopic microperimetry in the early diagnosis of age-related macular degeneration: preliminary study. BioMed Res Int. 2014;2014: 671529.

50 Crossland MD, Luong VA, Rubin GS, Fitzke FW. Retinal specific measurement of darkadapted visual function: validation of a modified microperimeter. BMC Ophthalmol. 2011 Feb;11(1):5.

51 Steinberg JS, Fitzke FW, Fimmers R, Fleckenstein M, Holz FG, Schmitz-Valckenberg S. Scotopic and photopic microperimetry in patients with reticular drusen and age-related macular degeneration. JAMA Ophthalmol. 2015 Jun; 133(6):690-7.

52 Pfau $M$, Lindner $M$, Fleckenstein $M$, Finger RP, Rubin GS, Harmening WM, et al. Testretest reliability of scotopic and mesopic fundus-controlled perimetry using a modified MAIA (Macular Integrity Assessment) in normal eyes. Ophthalmologica. 2017;237(1): 42-54.

53 Massof RW, Finkelstein D. Rod sensitivity relative to cone sensitivity in retinitis pigmentosa. Invest Ophthalmol Vis Sci. 1979 Mar; 18(3):263-72.

54 Heeren TF, Tzaridis S, Bonelli R, Pfau M, Fruttiger M, Okada M, et al. Dark-Adapted Two-Color Fundus-Controlled Perimetry in Macular Telangiectasia Type 2. Invest Ophthalmol Vis Sci. 2019 Apr;60(5):1760-7.

55 Pfau M, Lindner M, Gliem M, Steinberg JS, Thiele S, Finger RP, et al. Mesopic and darkadapted two-color fundus-controlled perimetry in patients with cuticular, reticular, and soft drusen. Eye. 2018 Dec;32(12):1819-30.

56 Norton TT, Corliss DA, Bailey JE. The psychophysical measurement of visual function. Volume 362. Mass, USA: Butterworth-Heinemann Boston; 2002.

57 Strauss RW, Kong X, Bittencourt MG, Ho A, Jha A, Schönbach EM, et al.; for the SMART Study Group. Scotopic Microperimetric Assessment of Rod Function in Stargardt Disease (SMART) Study: Design and Baseline Characteristics (Report No. 1). Ophthalmic Res. 2019;61(1):36-43.

58 von der Emde L, Pfau M, Thiele S, Möller PT, Hassenrik R, Fleckenstein M, et al. Mesopic and dark-adapted two-color fundus-controlled perimetry in choroidal neovascular- ization secondary to age-related macular degeneration. Transl Vis Sci Technol. 2018 Jan; $8(1): 7$.

59 Welker SG, Pfau M, Heinemann M, SchmitzValckenberg S, Holz FG, Finger RP. Retest reliability of mesopic and dark-adapted microperimetry in patients with intermediate agerelated macular degeneration and age-matched controls. Invest Ophthalmol Vis Sci. 2018 Mar;59(4):AMD152-9.

60 Pfau M, Müller PL, von der Emde L, Lindner M, Möller PT, Fleckenstein M, et al. Mesopic and dark-adapted two-color fundus-controlled perimetry in geographic atrophy secondary to age-related macular degeneration. Retina. 2020 Jan;40(1):169-80.

61 Han RC, Gray JM, Han J, Maclaren RE, Jolly JK. Optimisation of dark adaptation time required for mesopic microperimetry. $\mathrm{Br} \mathrm{J}$ Ophthalmol. 2019 Aug;103(8):1092-8.

62 Wong EN, Chew AL, Morgan WH, Patel PJ, Chen FK. The use of microperimetry to detect functional progression in non-neovascular age-related macular degeneration: a systematic review. Asia Pac J Ophthalmol. 2017 JanFeb;6(1):70-9.

63 Parodi MB, Triolo G, Morales M, Borrelli E, Cicinelli MV, Cascavilla ML, et al. MP1 and MAIA fundus perimetry in healthy subjects and patients affected by retinal dystrophies. Retina. 2015 Aug;35(8):1662-9.

64 Vujosevic S, Casciano M. Microperimetry: technical remarks. Microperimetry and Multimodal Retinal Imaging. Springer; 2014. pp. 13-22.

65 Wong EN, Mackey DA, Morgan WH, Chen FK. Inter-device comparison of retinal sensitivity measurements: the CenterVue MAIA and the Nidek MP-1. Clin Exp Ophthalmol. 2016 Jan-Feb;44(1):15-23.

66 Steinberg JS, Saßmannshausen M, Pfau M, Fleckenstein M, Finger RP, Holz FG, et al. Evaluation of two systems for fundus-controlled scotopic and mesopic perimetry in eye with age-related macular degeneration. Transl Vis Sci Technol. 2017 Jul;6(4):7.

67 Arango T, Morse AR, Seiple W. Comparisons of Two Microperimeters: The Clinical Value of an Extended Stimulus Range. Optom Vis Sci. 2018 Aug;95(8):663-71.

68 Balasubramanian S, Uji A, Lei J, Velaga S, Nittala M, Sadda S. Interdevice comparison of retinal sensitivity assessments in a healthy population: the CenterVue MAIA and the $\mathrm{Ni}$ dek MP-3 microperimeters. Br J Ophthalmol. 2018 Jan;102(1):109-13.

69 Artes PH, McLeod D, Henson DB. Response time as a discriminator between true- and false-positive responses in suprathreshold perimetry. Invest Ophthalmol Vis Sci. 2002 Jan; 43(1):129-32.

70 Greve EL. Single and multiple stimulus static perimetry in glaucoma: the two phases of perimetry. Springer; 1973. https://doi. org/10.1007/978-94-011-7765-8.

71 Olsson J, Bengtsson B, Heijl A, Rootzén H. An improved method to estimate frequency of false positive answers in computerized perim- etry. Acta Ophthalmol Scand. 1997 Apr; 75(2):181-3.

72 Heijl A, Krakau CE. An automatic static perimeter, design and pilot study. Acta Ophthalmol. 1975 Jun;53(3):293-310.

$73 \mathrm{Wu}$ Z, Ayton LN, Luu CD, Guymer RH. Longitudinal changes in microperimetry and low luminance visual acuity in age-related macular degeneration. JAMA Ophthalmol. 2015 Apr;133(4):442-8.

$74 \mathrm{Wu}$ Z, Ayton LN, Guymer RH, Luu CD. Intrasession test-retest variability of microperimetry in age-related macular degeneration. Invest Ophthalmol Vis Sci. 2013 Nov;54(12):7378-85.

75 Yamamoto S, et al. Microperimetry Shows Protection of Central Vision in Retinitis Pigmentosa Patients treated with UF-021: a Phase 2 Study (JapicCTI-090748). Invest Ophthalmol Vis Sci. 2011;52(14):4992.

76 Cassels NK, Wild JM, Margrain TH, Blyth C, Chong V, Acton JH. Microperimetry in agerelated macular degeneration: an evidencebase for pattern deviation probability analysis in microperimetry. Transl Vis Sci Technol. 2019 Dec;8(6):48.

77 Wu Z, Jung CJ, Ayton LN, Luu CD, Guymer RH. Test-retest repeatability of microperimetry at the border of deep scotomas. Invest Ophthalmol Vis Sci. 2015 Apr;56(4):2606-11.

78 Chen FK, Patel PJ, Xing W, Bunce C, Egan C, Tufail AT, et al. Test-retest variability of microperimetry using the Nidek MP1 in patients with macular disease. Invest Ophthalmol Vis Sci. 2009 Jul;50(7):3464-72.

79 Huang YM, Dou HL, Huang FF, Xu XR, Zou $\mathrm{ZY}, \mathrm{Lu} \mathrm{XR}$, et al. Changes following supplementation with lutein and zeaxanthin in retinal function in eyes with early age-related macular degeneration: a randomised, doubleblind, placebo-controlled trial. Br J Ophthalmol. 2015 Mar;99(3):371-5.

80 Vujosevic S, Bottega E, Casciano M, Pilotto E, Convento E, Midena E. Microperimetry and fundus autofluorescence in diabetic macular edema: subthreshold micropulse diode laser versus modified early treatment diabetic retinopathy study laser photocoagulation. Retina. 2010 Jun;30(6):908-16.

81 Vujosevic S, Martini F, Longhin E, Convento E, Cavarzeran F, Midena E. Subthreshold Micropulse Yellow Laser Versus Subthreshold Micropulse Infrared Laser in Center-Involving Diabetic Macular Edema: Morphologic and Functional Safety. Retina. 2015;35(8): 1594-603.

82 Comyn O, Sivaprasad S, Peto T, Neveu MM Holder GE, Xing W, et al. A randomized trial to assess functional and structural effects of ranibizumab versus laser in diabetic macular edema (the LUCIDATE study). Am J Ophthalmol. 2014 May;157(5):960-70.

83 Morescalchi F, Russo A, Bahja H, Gambicorti E, Cancarini A, Costagliola C, et al. FoveaSparing Versus Complete Internal Limiting Membrane Peeling in Vitrectomy for the Treatment of Macular Holes. Retina. $2020 \mathrm{Jul}$; 40(7):1306-14. 
84 Morescalchi F, Russo A, Gambicorti E, Cancarini A, Scaroni N, Bahja H, et al. Peeling of the Internal Limiting Membrane with Foveal Sparing for Treatment of Degenerative Lamellar Macular Hole. Retina. 2020 Jun;40(6): 1087-93.

85 Russo A, Morescalchi F, Gambicorti E, Cancarini A, Costagliola C, Semeraro F. Epiretinal Membrane Removal with Foveal-Sparing Internal Limiting Membrane Peeling: A Pilot Study. Retina. 2019 Nov;39(11):2116-24.

86 Eissa MG, Abdelhakim MA, Macky TA, Khafagy MM, Mortada HA. Functional and structural outcomes of ILM peeling in uncomplicated macula-off RRD using microperimetry \& en-face OCT. Graefes Arch Clin Exp Ophthalmol. 2018 Feb;256(2):249-57.

87 Ripandelli G, Scarinci F, Piaggi P, Guidi G, Pileri M, Cupo G, et al. Macular pucker: to peel or not to peel the internal limiting membrane? A microperimetric response. Retina. 2015 Mar;35(3):498-507.

88 Wong WT, Kam W, Cunningham D, Harrington $\mathrm{M}$, Hammel $\mathrm{K}$, Meyerle $\mathrm{CB}$, et al. Treatment of geographic atrophy by the topical administration of OT-551: results of a phase II clinical trial. Invest Ophthalmol Vis Sci. 2010 Dec;51(12):6131-9.

89 Wong WT, Dresner S, Forooghian F, Glaser T, Doss L, Zhou M, et al. Treatment of geographic atrophy with subconjunctival sirolimus: results of a phase I/II clinical trial. Invest Ophthalmol Vis Sci. 2013 Apr;54(4) 2941-50.

90 Petrou PA, Cunningham D, Shimel K, Harrington $\mathrm{M}$, Hammel $\mathrm{K}$, Cukras CA, et al. Intravitreal sirolimus for the treatment of geographic atrophy: results of a phase I/II clinical trial. Invest Ophthalmol Vis Sci. 2014 Dec; 56(1):330-8.

91 Hood DC, Anderson SC, Wall M, Kardon RH. Structure versus function in glaucoma: an application of a linear model. Invest Ophthalmol Vis Sci. 2007 Aug;48(8):3662-8.

92 Georgiou M, Singh N, Kane T, Zaman S, Hirji $\mathrm{N}$, Aboshiha J, et al. Long-Term Investigation of Retinal Function in Patients with Achromatopsia. Invest Ophthalmol Vis Sci. 2020 Sep;61(11):38-38.

93 Ochakovski GA, et al. Effects of Subretinal AAV8 Gene Therapy on Microperimetry in CNGA3 Achromatopsia Patients. Invest Ophthalmol Vis Sci. 2019;60(9):2921.

94 Mehat MS, Sundaram V, Ripamonti C, Robson AG, Smith AJ, Borooah S, et al. Transplantation of Human Embryonic Stem CellDerived Retinal Pigment Epithelial Cells in Macular Degeneration. Ophthalmology. 2018 Nov;125(11):1765-75.

95 Bland JM, Altman DG. Agreement between methods of measurement with multiple observations per individual. J Biopharm Stat. 2007;17(4):571-82.

96 Dow CT, Harley CB. Evaluation of an oral telomerase activator for early age-related macular degeneration - a pilot study. Clin Ophthalmol. 2016 Jan;10:243-9.
97 Dunavoelgyi R, Sacu S, Simader C, Pruente C, Schmidt-Erfurth U. Changes in macular sensitivity after reduced fluence photodynamic therapy combined with intravitreal triamcinolone. Acta Ophthalmol. 2011 Mar;89(2):166-71.

98 Heier JS, Pieramici D, Chakravarthy U, Patel SS, Gupta S, Lotery A, et al.; Chroma and Spectri Study Investigators. Visual Function Decline Resulting from Geographic Atrophy: Results from the Chroma and Spectri Phase 3 Trials. Ophthalmol Retina. 2020 Jul; $4(7): 673-88$.

99 Wong EN, De Soyza JD, Mackey DA, Constable IJ, Chen FK. Intersession test-retest variability of microperimetry in type 2 macular telangiectasia. Transl Vis Sci Technol. 2017 Dec;6(6):7.

100 Fujii GY, de Juan E Jr, Sunness J, Humayun MS, Pieramici DJ, Chang TS. Patient selection for macular translocation surgery using the scanning laser ophthalmoscope. Ophthalmology. 2002 Sep;109(9):1737-44.

101 Steinman RM. Effect of target size, luminance, and color on monocular fixation. JOSA. 1965;55(9):1158-64.

102 Crossland MD, Dunbar HM, Rubin GS. Fixation stability measurement using the MP1 microperimeter. Retina. 2009 May;29(5):651-6.

103 Grenga PL, Fragiotta S, Meduri A, Lupo S, Marenco M, Vingolo EM. Fixation stability measurements in patients with neovascular age-related macular degeneration treated with ranibizumab. Can J Ophthalmol. 2013 Oct;48(5):394-9.

104 Schönbach EM, Ibrahim MA, Kong X, Strauss RW, Muñoz B, Birch DG, et al. Metrics and Acquisition Modes for Fixation Stability as a Visual Function Biomarker. Invest Ophthalmol Vis Sci. 2017 May; 58(6):BIO268-76.

105 Schönbach EM, Strauss RW, Kong X, Muñoz B, Ibrahim MA, Sunness JS, et al.; ProgStar Study Group. Longitudinal Changes of Fixation Location and Stability Within 12 Months in Stargardt Disease: ProgStar Report No. 12. Am J Ophthalmol. 2018 Sep; 193:54-61.

106 Schönbach EM, Ibrahim MA, Strauss RW, Birch DG, Cideciyan AV, Hahn GA, et al.; Progression of Stargardt Disease Study Group. Fixation Location and Stability Using the MP-1 Microperimeter in Stargardt Disease: ProgStar Report No. 3. Ophthalmol Retina. 2017 Jan - Feb;1(1):68-76.

107 MacLaren RE, Groppe M, Barnard AR, Cottriall CL, Tolmachova T, Seymour L, et al. Retinal gene therapy in patients with choroideremia: initial findings from a phase $1 / 2$ clinical trial. Lancet. 2014 Mar;383(9923): 1129-37.

108 Jolly JK, Edwards TL, Moules J, Groppe M, Downes SM, MacLaren RE. A Qualitative and Quantitative Assessment of Fundus Autofluorescence Patterns in Patients With Choroideremia. Invest Ophthalmol Vis Sci. 2016 Aug;57(10):4498-503.
109 Fischer MD, Ochakovski GA, Beier B, Seitz IP, Vaheb Y, Kortuem C, et al. CHANGES in RETINAL SENSITIVITY after GENE THERAPY in CHOROIDEREMIA. Retina. 2020 Jan;40(1):160-8.

110 Bainbridge JW, Mehat MS, Sundaram V, Robbie SJ, Barker SE, Ripamonti C, et al. Long-term effect of gene therapy on Leber's congenital amaurosis. N Engl J Med. 2015 May;372(20):1887-97.

111 Bainbridge JW, Smith AJ, Barker SS, Robbie S, Henderson R, Balaggan K, et al. Effect of gene therapy on visual function in Leber's congenital amaurosis. N Engl J Med. 2008 May;358(21):2231-9.

112 Hauswirth WW, Aleman TS, Kaushal S, Cideciyan AV, Schwartz SB, Wang L, et al. Treatment of leber congenital amaurosis due to RPE65 mutations by ocular subretinal injection of adeno-associated virus gene vector: short-term results of a phase I trial. Hum Gene Ther. 2008 Oct;19(10):979-90.

113 Jacobson SG, Cideciyan AV, Roman AJ, Sumaroka A, Schwartz SB, Heon E, et al. Improvement and decline in vision with gene therapy in childhood blindness. $\mathrm{N}$ Engl J Med. 2015 May;372(20):1920-6.

114 Maguire AM, High KA, Auricchio A, Wright JF, Pierce EA, Testa F, et al. Agedependent effects of RPE65 gene therapy for Leber's congenital amaurosis: a phase 1 dose-escalation trial. Lancet. 2009 Nov; 374(9701):1597-605

115 Maguire AM, Simonelli F, Pierce EA, Pugh EN Jr, Mingozzi F, Bennicelli J, et al. Safety and efficacy of gene transfer for Leber's congenital amaurosis. N Engl J Med. 2008 May; 358(21):2240-8.

116 Bennett J, Wellman J, Marshall KA, McCague S, Ashtari M, DiStefano-Pappas J, et al. Safety and durability of effect of contralateral-eye administration of AAV2 gene therapy in patients with childhood-onset blindness caused by RPE65 mutations: a follow-on phase 1 trial. Lancet. 2016 Aug; 388(10045):661-72.

117 Russell S, Bennett J, Wellman JA, Chung DC, Yu ZF, Tillman A, et al. Efficacy and safety of voretigene neparvovec (AAV2hRPE65v2) in patients with RPE65-mediated inherited retinal dystrophy: a randomised, controlled, open-label, phase 3 trial. Lancet. 2017 Aug;390(10097):849-60.

118 Le Meur G, Lebranchu P, Billaud F, Adjali O, Schmitt S, Bézieau S, et al. Safety and long-term efficacy of AAV4 gene therapy in patients with RPE65 Leber congenital amaurosis. Mol Ther. 2018 Jan;26(1):256-68.

119 Weleber RG, Pennesi ME, Wilson DJ, Kaushal S, Erker LR, Jensen L, et al. Results at 2 years after gene therapy for RPE65-deficient Leber congenital amaurosis and severe early-childhood-onset retinal dystrophy. Ophthalmology. 2016 Jul;123(7):1606-20.

120 Bainbridge J, Ali R. Gene therapy for inherited childhood blindness shows promise. Expert Rev Ophthalmol. 2008;3(4):357-9. 
121 Georgiadis A, Duran Y, Ribeiro J, AbelleiraHervas L, Robbie SJ, Sünkel-Laing B, et al. Development of an optimized AAV2/5 gene therapy vector for Leber congenital amaurosis owing to defects in RPE65. Gene Ther. 2016 Dec;23(12):857-62.

122 Testa F, Maguire AM, Rossi S, Marshall K, Auricchio A, Melillo P, et al. Evaluation of ocular gene therapy in an italian patient affected by congenital leber amaurosis type 2 treated in both eyes. Adv Exp Med Biol. 2016;854:533-9.

123 AGTC. XLRP phase 1/2 clinical trial updated preliminary interim data. Applied Genetic Technologies Corporation; 2020. http://ir.agtc.com/static-files/254008cf6089-4009-ad63-808d7044a7ef (accessed Dec 21, 2020).

124 AGTC. AGTC reports positive six-month data from its ongoing phase $1 / 2$ clinical trial in X-linked retinitis pigmentosa. Applied Genetic Technologies Corporation; 2020. https://agtc.com/agtc-reports-positive-sixmonth-data-from-its-ongoing-phase1-2-clinical-trial-in-x-linked-retinitis-pigmentosa/ (accessed Dec 21, 2020).

125 AGTC. AGTC reports additional positive data from its phase $1 / 2$ clinical trial in patients with X-linked retinitis pigmentosa. Applied Genetic Technologies Corporation; 2020. http://ir.agtc.com/news-releases/news-release-details/agtc-reports-additional-positive-data-its-phase-12-clinical (accessed Dec 21, 2020).

126 Fischer MD, Michalakis S, Wilhelm B, Zobor D, Muehlfriedel R, Kohl S, et al. Safety and Vision Outcomes of Subretinal Gene Therapy Targeting Cone Photoreceptors in Achromatopsia: A Nonrandomized Controlled Trial. JAMA Ophthalmol. 2020 Jun; 138(6):643-51.

127 Kong X, Strauss RW, Michaelides M, Cideciyan AV, Sahel JA, Muñoz B, et al.; ProgStar Study Group. Visual acuity loss and associated risk factors in the retrospective progression of stargardt disease study (ProgStar Report No. 2). Ophthalmology. 2016 Sep;123(9):1887-97.

128 Fujinami K, Lois N, Mukherjee R, McBain VA, Tsunoda K, Tsubota K, et al. A longitudinal study of Stargardt disease: quantitative assessment of fundus autofluorescence, progression, and genotype correlations. Invest Ophthalmol Vis Sci. 2013 Dec;54(13): 8181-90.

129 Schönbach EM, Strauss RW, Ibrahim MA, Janes JL, Birch DG, Cideciyan AV, et al.; ProgStar Study Group. Faster Sensitivity Loss around Dense Scotomas than for Overall Macular Sensitivity in Stargardt Disease: ProgStar Report No. 14. Am J Ophthalmol. 2020 Aug;216:219-25.

130 Schönbach EM, Wolfson Y, Strauss RW, Ibrahim MA, Kong X, Muñoz B, et al.; ProgStar Study Group. (ProgStar) Study: report No. 7. JAMA Ophthalmol. 2017 Jul;135(7): 696-703.
131 Schonbach EM. Month 24 results from the scotopic microperimetric assessment of rod function in Stargardt diseasedisease (SMART) study. American Academy of Ophthalmology Annual Meeting. 2019.

132 Ervin AM, Strauss RW, Ahmed MI, Birch D, Cheetham J, Ferris FL 3rd, et al.; ProgStar Study Group. A Workshop on Measuring the Progression of Atrophy Secondary to Stargardt Disease in the ProgStar Studies: Findings and Lessons Learned. Transl Vis Sci Technol. 2019 Apr;8(2):16.

133 Salvatore S, Fishman GA, McAnany JJ, Genead MA. Association of dark-adapted visual function with retinal structural changes in patients with Stargardt disease. Retina. 2014 May;34(5):989-95.

134 Strauss RW, Muñoz B, Ahmed MI, Bittencourt M, Schönbach EM, Michaelides M, et al.; for the ProgStar-4 Study Group. The Progression of the Stargardt Disease Type 4 (ProgStar-4) Study: Design and Baseline Characteristics (ProgStar-4 Report No. 1). Ophthalmic Res. 2018;60(3):185-94.

135 Cassels NK, Wild JM, Margrain TH, Chong $\mathrm{V}$, Acton $\mathrm{JH}$. The use of microperimetry in assessing visual function in age-related macular degeneration. Surv Ophthalmol. 2018 Jan - Feb;63(1):40-55.

136 Midena E, Pilotto E. Microperimetry in age: related macular degeneration. Eye. 2017 Jul; 31(7):985-94.

137 Markowitz SN, Reyes SV. Microperimetry and clinical practice: an evidence-based review. Can J Ophthalmol. 2013 Oct;48(5): $350-7$.

138 Sunness JS, Schuchard RA, Shen N, Rubin GS, Dagnelie G, Haselwood DM. Landmarkdriven fundus perimetry using the scanning laser ophthalmoscope. Invest Ophthalmol Vis Sci. 1995 Aug;36(9):1863-74.

139 Wu Z, Ayton LN, Guymer RH, Luu CD. Low-luminance visual acuity and microperimetry in age-related macular degeneration. Ophthalmology. 2014 Aug;121(8): 1612-9.

140 Chandramohan A, Stinnett SS, Petrowski JT, Schuman SG, Toth CA, Cousins SW, et al. Visual function measures in early and intermediate age-related macular degeneration. Retina. 2016 May;36(5):1021-31.

141 Parisi V, Perillo L, Tedeschi M, Scassa C, Gallinaro G, Capaldo N, et al. Macular function in eyes with early age-related macular degeneration with or without contralateral late age-related macular degeneration. Retina. 2007 Sep;27(7):879-90.

142 Vujosevic S, Smolek MK, Lebow KA, Notaroberto N, Pallikaris A, Casciano M. Detection of macular function changes in early (AREDS 2) and intermediate (AREDS 3) age-related macular degeneration. Ophthalmologica. 2011;225(3):155-60.

143 Saßmannshausen M, Zhou J, Pfau M, Thiele S, Steinberg J, Fleckenstein M, et al. Longitudinal analysis of retinal thickness and retinal function in eyes with large drusen sec- ondary to intermediate age-related macular degeneration. Ophthalmol Retina. 2020, Online ahead of print.

144 Sassmannshausen M, Pfau M, Thiele S, Fimmers R, Steinberg JS, Fleckenstein M, et al. Longitudinal Analysis of Structural and Functional Changes in Presence of Reticular Pseudodrusen Associated With Age-Related Macular Degeneration. Invest Ophthalmol Vis Sci. 2020 Aug;61(10):19.

145 Hsu ST, Thompson AC, Stinnett SS, Luhmann UF, Vajzovic L, Horne A, et al.; Longitudinal Study of Visual Function in Dry Age-Related Macular Degeneration at 12 Months. Longitudinal Study of Visual Function in Dry Age-Related Macular Degeneration at 12 Months. Ophthalmol Retina. 2019 Aug;3(8):637-48.

146 Weigert G, Kaya S, Pemp B, Sacu S, Lasta M, Werkmeister RM, et al. Effects of lutein supplementation on macular pigment optical density and visual acuity in patients with agerelated macular degeneration. Invest Ophthalmol Vis Sci. 2011 Oct;52(11):8174-8.

147 Sacu S, Varga A, Michels S, Weigert G, Polak K, Vécsei-Marlovits PV, et al. Reduced fluence versus standard photodynamic therapy in combination with intravitreal triamcinolone: short-term results of a randomised study. Br J Ophthalmol. 2008 Oct; 92(10):1347-51.

148 Rezar-Dreindl S, Eibenberger K, Buehl W, Georgopoulos M, Weigert G, Krall C, et al. Role of additional dexamethasone for the management of persistent or recurrent neovascular agerelated macular degeneration under ranibizumab treatment. Retina. 2017 May;37(5):962-70.

149 Chew EY, Clemons TE, Jaffe GJ, Johnson CA, Farsiu S, Lad EM, et al.; Macular Telangiectasia Type 2-Phase 2 CNTF Research Group. Effect of Ciliary Neurotrophic Factor on Retinal Neurodegeneration in $\mathrm{Pa}$ tients with Macular Telangiectasia Type 2: A Randomized Clinical Trial. Ophthalmology. 2019 Apr;126(4):540-9.

150 Wu Z, Luu CD, Hodgson LA, Caruso E, Brassington $\mathrm{KH}$, Tindill N, et al.; LEAD Study Group. Secondary and Exploratory Outcomes of the Subthreshold Nanosecond Laser Intervention Randomized Trial in Age-Related Macular Degeneration: A LEAD Study Report. Ophthalmol Retina. 2019 Dec;3(12):1026-34.

151 Rinaldi M, Semeraro F, Chiosi F, Russo A, Romano MR, Savastano MC, et al. Reducedfluence verteporfin photodynamic therapy plus ranibizumab for choroidal neovascularization in pathologic myopia. Graefes Arch Clin Exp Ophthalmol. 2017 Mar; 255(3):529-39.

152 Limoli, P.G., E.M. Vingolo, C. Limoli, S.Z. Scalinci, and M. Nebbioso, Regenerative Therapy by Suprachoroidal Cell Autograft in Dry Age-related Macular Degeneration: Preliminary In Vivo Report. J Vis Exp. 2018 Feb;(132):56469. 
153 Sallo FB, Peto T, Egan C, Wolf-Schnurrbusch UE, Clemons TE, Gillies MC, et al.; MacTel Study Group. "En face" OCT imaging of the IS/OS junction line in type 2 idiopathic macular telangiectasia. Invest Ophthalmol Vis Sci. 2012 Sep;53(10):6145-52.

154 Sallo FB, Leung I, Clemons TE, Peto T, Chew EY, Pauleikhoff D, et al.; MacTel CNTF Research Group. Correlation of Structural and Functional Outcome Measures in a Phase One Trial of Ciliary Neurotrophic Factor in Type 2 Idiopathic Macular Telangiectasia. Retina. 2018 Jan;38 Suppl 1:S27-32.

155 Kandasamy R, Wickremasinghe S, Guymer R. New treatment modalities for geographic atrophy. Asia Pac J Ophthalmol. 2017 NovDec;6(6):508-13.

156 Meleth AD, Mettu P, Agrón E, Chew EY, Sadda SR, Ferris FL, et al. Changes in retinal sensitivity in geographic atrophy progression as measured by microperimetry. Invest Ophthalmol Vis Sci. 2011 Feb;52(2):1119-26.

157 Wu Z, Cimetta R, Caruso E, Guymer RH. Performance of a defect-mapping microperimetry approach for characterizing progressive changes in deep scotomas. Transl Vis Sci Technol. 2019 Aug;8(4):16.

158 Mitchell P, Bandello F, Schmidt-Erfurth U, Lang GE, Massin P, Schlingemann RO, et al.; RESTORE study group. The RESTORE study: ranibizumab monotherapy or combined with laser versus laser monotherapy for diabetic macular edema. Ophthalmology. 2011 Apr;118(4):615-25.

159 Massin P, Bandello F, Garweg JG, Hansen LL, Harding SP, Larsen M, et al. Safety and efficacy of ranibizumab in diabetic macular edema (RESOLVE Study): a 12-month, randomized, controlled, double-masked, multicenter phase II study. Diabetes Care. 2010 Nov;33(11):2399-405.

160 Korobelnik JF, Do DV, Schmidt-Erfurth U, Boyer DS, Holz FG, Heier JS, et al. Intravitreal aflibercept for diabetic macular edema. Ophthalmology. 2014 Nov;121(11):2247-54.

161 Rajendram R, Fraser-Bell S, Kaines A, Michaelides M, Hamilton RD, Esposti SD, et al. A 2-year prospective randomized controlled trial of intravitreal bevacizumab or laser therapy (BOLT) in the management of diabetic macular edema: 24-month data: report 3. Arch Ophthalmol. 2012 Aug;130(8):972-9.

162 Nguyen QD, Brown DM, Marcus DM, Boyer DS, Patel S, Feiner L, et al.; RISE and RIDE Research Group. Ranibizumab for diabetic macular edema: results from 2 phase III randomized trials: RISE and RIDE. Ophthalmology. 2012 Apr;119(4):789-801.

163 Early Treatment Diabetic Retinopathy Study Research Group. Early photocoagulation for diabetic retinopathy. ETDRS report number 9. Ophthalmology. 1991 May;98(5 Suppl):766-85.

164 Gonzalez VH, Boyer DS, Schmidt-Erfurth U, Heier JS, Gordon C, Benz MS, et al. Microperimetric assessment of retinal sensitivity in eyes with diabetic macular edema from a phase 2 study of intravitreal aflibercept. Retina. 2015 Apr;35(4):687-94.

165 Mylonas G, Schranz M, Scholda C, Karst S, Reiter G, Baumann L, et al. Response of Retinal Sensitivity to Intravitreal Anti-angiogenic Bevacizumab and Triamcinolone Acetonide for Patients with Diabetic Macular Edema over One Year. Curr Eye Res. 2020 Sep;45(9):1107-13.

166 Do DV, Nguyen QD, Boyer D, Schmidt-Erfurth U, Brown DM, Vitti R, et al.; da Vinci Study Group. One-year outcomes of the da Vinci Study of VEGF Trap-Eye in eyes with diabetic macular edema. Ophthalmology. 2012 Aug;119(8):1658-65.

167 Kriechbaum K, Prager S, Mylonas G, Scholda C, Rainer G, Funk M, et al.; Diabetic Retinopathy Research Group. Intravitreal bevacizumab (Avastin) versus triamcinolone (Volon A) for treatment of diabetic macular edema: one-year results. Eye. 2014 Jan;28(1):9-15.

168 Valent DJ, Wong WT, Chew EY, Cukras CA. Oral dextromethorphan for the treatment of diabetic macular edema: results from a phase I/II clinical study. Transl Vis Sci Technol. 2018 Dec;7(6):24.

169 Lois N, Gardner E, McFarland M, Armstrong D, McNally C, Lavery NJ, et al. A phase 2 clinical trial on the use of cibinetide for the treatment of diabetic macular edema. J Clin Med. 2020 Jul;9(7):1-14.

170 Gella L, Raman R, Kulothungan V, Saumya Pal S, Ganesan S, Sharma T. Retinal sensitivity in subjects with type 2 diabetes mellitus: sankara Nethralaya diabetic retinopathy epidemiology and molecular genetics study (SN-DREAMS II, Report no. 4). Br J Ophthalmol. 2016 Jun;100(6):808-13.

171 Rodríguez González-Herrero ME, Ruiz M, López Román FJ, Marín Sánchez JM, Domingo JC. Supplementation with a highly concentrated docosahexaenoic acid plus xanthophyll carotenoid multivitamin in nonproliferative diabetic retinopathy: prospective controlled study of macular function by fundus microperimetry. Clin Ophthalmol. 2018 May;12:1011-20.

172 Longhin E, Tormene AP, Olivato E, Convento E, Vujosevic S, Pilotto E, et al. Rod function in diabetic patients without and with early diabetic retinopathy. Eur J Ophthalmol. 2016 Aug;26(5):418-24.

173 van Dijk EH, Fauser S, Breukink MB, Blanco-Garavito R, Groenewoud JM, Keunen JE, et al. Half-Dose Photodynamic Therapy versus High-Density Subthreshold Micropulse Laser Treatment in Patients with Chronic Central Serous Chorioretinopathy: the PLACE Trial. Ophthalmology. 2018 Oct;125(10):1547-55.

174 van Rijssen TJ, van Dijk EH, Scholz P, Breukink MB, Dijkman G, Peters PJ, et al. Crossover to Photodynamic Therapy or Micropulse Laser After Failure of Primary Treatment of Chronic Central Serous Chorioretinopathy: the REPLACE Trial. Am J Ophthalmol. 2020 Aug;216:80-9.
175 Dang Y, Mu Y, Zhao M, Li L, Guo Y, Zhu Y The effect of eradicating Helicobacter pylori on idiopathic central serous chorioretinopathy patients. Ther Clin Risk Manag. 2013;9(1):355-60.

176 Mangione CM, Lee PP, Gutierrez PR Spritzer K, Berry S, Hays RD; National Eye Institute Visual Function Questionnaire Field Test Investigators. Development of the 25-list-item national eye institute visual function questionnaire. Arch Ophthalmol. 2001 Jul;119(7):1050-8.

177 Viana KI, et al. Combined pars plana vitrectomy (PPV) and phacoemulsification (phaco) versus PPV and deferred phaco for phakic patients with full-thickness macular hole (FTMH) and no significant cataract at baseline: 1-year outcomes of a randomized trial combined $\mathrm{PPV} /$ phaco vs $\mathrm{PPV} /$ deferred phaco for MH. Graefes Arch Clin Exp Ophthalmol. 2021 Jan;259(1):29-36.

178 Romano MR, Cennamo G, Grassi P, Sparnelli F, Allegrini D, Cennamo G. Changes in macular pigment optical density after membrane peeling [Electronic Resource]. PLoS One. 2018 May;13(5):e0197034.

179 Wallsh J, Sharareh B, Gallemore R. Therapeutic effect of dexamethasone implant in retinal vein occlusions resistant to antiVEGF therapy. Clin Ophthalmol. 2016 May; 10:947-54.

180 Pfau M, Lindner M, Steinberg JS, Thiele S, Brinkmann CK, Fleckenstein M, et al. Visual field indices and patterns of visual field deficits in mesopic and dark-adapted two-colour fundus-controlled perimetry in macular diseases. Br J Ophthalmol. 2018 Aug;102(8):1054-9.

181 Katz J. A comparison of the pattern- and total deviation-based Glaucoma Change Probability programs. Invest Ophthalmol Vis Sci. 2000 Apr;41(5):1012-6.

182 Weinreb RN, Kaufman PL. The glaucoma research community and FDA look to the future: a report from the NEI/FDA CDER Glaucoma Clinical Trial Design and Endpoints Symposium. Invest Ophthalmol Vis Sci. 2009 Apr;50(4):1497-505.

183 von der Emde L, Pfau M, Dysli C, Thiele S, Möller PT, Lindner M, et al. Artificial intelligence for morphology-based function prediction in neovascular age-related macular degeneration. Sci Rep. 2019 Jul;9(1):11132.

184 Kihara Y, Heeren TF, Lee CS, Wu Y, Xiao S, Tzaridis S, et al. Estimating Retinal Sensitivity Using Optical Coherence Tomography With Deep-Learning Algorithms in Macular Telangiectasia Type 2. JAMA Netw Open. 2019 Feb;2(2):e188029.

185 Schonbach EM, et al. Longitudinal Microperimetric Changes of Macular Sensitivity in Stargardt Disease after 12 Months: ProgStar Report No. 13. JAMA Ophthalmol. 2020 Jul;138(7):772-9.

186 Tan CS, Sadda SR. The role of central reading centers - current practices and future directions. Indian J Ophthalmol. 2015 May; 63(5):404-5. 
187 Squirrell DM, Mawer NP, Mody CH, Brand CS. Visual outcome after intravitreal ranibizumab for wet age-related macular degeneration: a comparison between best-corrected visual acuity and microperimetry. Retina. 2010 Mar;30(3):436-42.

188 Jeffrey BG, Cukras CA, Vitale S, Turriff A Bowles K, Sieving PA. Test-retest intervisit variability of functional and structural parameters in X-linked retinoschisis. Transl Vis Sci Technol. 2014 Oct;3(5):5.

189 Buckley TM, Jolly JK, Menghini M, Wood LJ, Nanda A, MacLaren RE. Test-retest repeatability of microperimetry in patients with retinitis pigmentosa caused by mutations in RPGR. Clin Exp Ophthalmol. 2020 Jul;48(5):714-5.

190 Cideciyan AV, Swider M, Aleman TS, Feuer WJ, Schwartz SB, Russell RC, et al. Macular function in macular degenerations: repeatability of microperimetry as a potential outcome measure for ABCA4-associated retinopathy trials. Invest Ophthalmol Vis Sci. $2012 \mathrm{Feb} ; 53(2): 841-52$.

191 Alibhai AY, Mehta N, Hickson-Curran S, Moreira-Neto C, Levine ES, Reichel E, et al. Test-retest variability of microperimetry in geographic atrophy. Int J Retina Vitreous. 2020 Apr;6(1):16.

192 Jones PR, Yasoubi N, Nardini M, Rubin GS Feasibility of macular integrity assessment (Maia) microperimetry in children: Sensitivity, reliability, and fixation stability in healthy observers. Invest Ophthalmol Vis Sci. 2016 Nov;57(14):6349-59.

193 Sankoh AJ, Li H, D’Agostino RB Sr. Use of composite endpoints in clinical trials. Stat Med. 2014 Nov;33(27):4709-14.

194 Medeiros FA, Lisboa R, Weinreb RN, Girkin CA, Liebmann JM, Zangwill LM. A combined index of structure and function for staging glaucomatous damage. Arch Ophthalmol. 2012 Sep;130(9):1107-16.

195 Montesano G, Rossetti LM, McKendrick AM, Turpin A, Fogagnolo P, Oddone F, et al. Effect of fundus tracking on structurefunction relationship in glaucoma. $\mathrm{Br}$ Ophthalmol. 2020 Dec;104(12):1710-6.

196 Lambertus, S., et al., Highly sensitive measurements of disease progression in rare disorders: Developing and validating a multimodal model of retinal degeneration in Stargardt disease. PLoS One. 2017; 12(3): 0174020

197 Schliesser JA, Gallimore G, Kunjukunju N, Sabates NR, Koulen P, Sabates FN. Clinical application of optical coherence tomography in combination with functional diagnostics: advantages and limitations for diagnosis and assessment of therapy outcome in central serous chorioretinopathy. Clin Ophthalmol. 2014 Nov; 8:2337-45.

$198 \mathrm{Wu}$ Z, Luu CD, Hodgson LA, Caruso E, Chen FK, Chakravarthy U, et al. Examining the added value of microperimetry and low luminance deficit for predicting progres- sion in age-related macular degeneration. Br J Ophthalmol. 2020, Online ahead of print.

199 Wu Z, Cunefare D, Chiu E, Luu CD, Ayton LN, Toth CA, et al. Longitudinal associations between microstructural changes and microperimetry in the early stages of agerelated macular degeneration. Invest Ophthalmol Vis Sci. 2016 Jul;57(8):3714-22.

$200 \mathrm{Wu}$ Z, Ayton LN, Luu CD, Guymer RH. Relationship between retinal microstructures on optical coherence tomography and microperimetry in age-related macular degeneration. Ophthalmology. 2014 Jul;121(7): 1445-52.

201 Wu Z, Luu CD, Hodgson LA, Caruso E, Tindill N, Aung KZ, et al. Prospective longitudinal evaluation of nascent geographic atrophy in age-related macular degeneration. Ophthalmol Retina. 2020 Jun;4(6): 568-75.

202 Yohannan J, Bittencourt M, Sepah YJ, Hatef E, Sophie R, Moradi A, et al. Association of retinal sensitivity to integrity of photoreceptor inner/outer segment junction in patients with diabetic macular edema. Ophthalmology. 2013 Jun;120(6):1254-61.

203 Meleth AD, Toy BC, Nigam D, Agrón E, Murphy RP, Chew EY, et al. Prevalence and progression of pigment clumping associated with idiopathic macular telangiectasia type 2. Retina. 2013 Apr;33(4):762-70

204 Mukherjee D, Lad EM, Vann RR, Jaffe SJ, Clemons TE, Friedlander M, et al.; MacTel Study Group. Correlation Between Macular Integrity Assessment and Optical Coherence Tomography Imaging of Ellipsoid Zone in Macular Telangiectasia Type 2. Invest Ophthalmol Vis Sci. 2017 May; 58(6):BIO291-9.

205 Fischer MD, Ochakovski GA, Beier B, Seitz IP, Vaheb Y, Kortuem C, et al. Efficacy and Safety of Retinal Gene Therapy Using Adeno-Associated Virus Vector for Patients With Choroideremia: A Randomized Clinical Trial. JAMA Ophthalmol. 2019 Aug; 137(11):1247-54

206 Lenassi E, Troeger E, Wilke R, Tufail A, Hawlina M, Jeffery G, et al. Laser clearance of drusen deposit in patients with autosomal dominant drusen (p.Arg345Trp in EFEMP1). Am J Ophthalmol. 2013 Jan; 155(1):190-8

207 Yamamoto S, Sugawara T, Murakami A, Nakazawa M, Nao-I N, Machida S, et al. Topical isopropyl unoprostone for retinitis pigmentosa: microperimetric results of the phase 2 clinical study. Ophthalmol Ther. 2012 Dec;1(1):5.

208 Tawada A, Sugawara T, Ogata K, Hagiwara A, Yamamoto S. Improvement of central retinal sensitivity six months after topical isopropyl unoprostone in patients with retinitis pigmentosa. Indian J Ophthalmol. 2013 Mar;61(3):95-9.

209 Wagner SK, Jolly JK, Pefkianaki M, Gekeler F, Webster AR, Downes SM, et al. Transcor- neal electrical stimulation for the treatment of retinitis pigmentosa: results from the TESOLAUK trial. BMJ Open Ophthalmol. 2017 Dec;2(1):e000096.

210 Campochiaro PA, Iftikhar M, Hafiz G, Akhlaq A, Tsai G, Wehling D, et al. Oral Nacetylcysteine improves cone function in retinitis pigmentosa patients in phase I trial. J Clin Invest. 2020 Mar;130(3):1527-41.

211 Kong X, Hafiz G, Wehling D, Akhlaq A, Campochiaro PA. Locus Level Changes in Macular Sensitivity in Patients with Retinitis Pigmentosa Treated with Oral N-acetylcysteine. Am J Ophthalmol. 2021 Jan;221: 105-14.

212 Chen FK, Uppal GS, Rubin GS, Webster AR, Coffey PJ, Da Cruz L. Evidence of retinal function using microperimetry following autologous retinal pigment epithelium-choroid graft in macular dystrophy. Invest Ophthalmol Vis Sci. 2008 Jul;49(7):3143-50.

213 Park SS, Bauer G, Abedi M, Pontow S, Panorgias A, Jonnal R, et al. Intravitreal autologous bone marrow $\mathrm{CD} 34+$ cell therapy for ischemic and degenerative retinal disorders: preliminary phase 1 clinical trial findings. Invest Ophthalmol Vis Sci. 2014 Dec;56(1): 81-9.

214 Do DV, Schmidt-Erfurth U, Gonzalez VH, Gordon CM, Tolentino M, Berliner AJ, et al. The DA VINCI Study: phase 2 primary results of VEGF Trap-Eye in patients with diabetic macular edema. Ophthalmology. 2011 Sep;118(9):1819-26.

215 Forte R, Cennamo G, Finelli ML, Bonavolontà $\mathrm{P}$, de Crecchio G, Greco GM. Combination of flavonoids with Centella asiatica and Melilotus for diabetic cystoid macular edema without macular thickening. J Ocul Pharmacol Ther. 2011 Apr; 27(2):109-13.

216 Mackensen F, Jakob E, Springer C, Dobner $\mathrm{BC}$, Wiehler $\mathrm{U}$, Weimer $\mathrm{P}$, et al. Interferon versus methotrexate in intermediate uveitis with macular edema: results of a randomized controlled clinical trial. Am J Ophthalmol. 2013 Sep;156(3):478-86.e1

217 Breukink MB, Downes SM, Querques G, van Dijk EH, den Hollander AI, Blanco-Garavito $\mathrm{R}$, et al. Comparing half-dose photodynamic therapy with high-density subthreshold micropulse laser treatment in patients with chronic central serous chorioretinopathy (the PLACE trial): study protocol for a randomized controlled trial. Trials. 2015 Sep;16(1):419.

218 Romano MR, Ilardi G, Ferrara M, Cennamo G, Parolini B, Mariotti C, et al. Macular peeling-induced retinal damage: clinical and histopathological evaluation after using different dyes. Graefes Arch Clin Exp Ophthalmol. 2018 Sep;256(9):1573-80.

219 Pilotto E, Urban F, Parrozzani R, Midena E. Standard versus bolus photodynamic therapy in circumscribed choroidal hemangioma: functional outcomes. Eur J Ophthalmol. 2011 Jul-Aug;21(4):452-8. 